\title{
Quality Control of Binary Distillation Columns via Nonlinear Aggregated Models*
}

\author{
J. LÉVINE $\dagger$ and P. ROUCHON $\ddagger$
}

\begin{abstract}
A robust nonlinear control law is designed to reject unknown feed composition disturbances with overall stability. Implementation to real columns as well as comparisons with classical control strategies, show the robustness and flexibility improvements of the method.
\end{abstract}

Key Words-Control applications; distillation columns; disturbance rejection; model reduction; nonlinear control systems; overall stability; quality control; singular perturbations.

\begin{abstract}
Using singular perturbation techniques on a physical model of distillation column, an aggregated model is proposed for control purposes. Nonlinear perturbation rejection techniques via static feedback are then applied to this model to reject the feed composition disturbances: around every slowly varying reference trajectory, the existence of such a control law and the stability of the overall closed-loop system are proven. Moreover, the obtained control law can be synthesized with measurements commonly available on distillation columns: the product compositions and two inner temperatures. An industrial implementation on a refinery depropanizer of 42 trays is presented. Simulation comparisons with linear and nonlinear geometric control laws, both using the physical model of high dimension, show the robustness and flexibility improvements provided by our method.
\end{abstract}

\section{Notation}

$f=\left(f_{j}\right)_{j=1, \ldots, n}:$ state function of the physical model (1).

$f=\left(f_{j}\right)_{j=1, r, j}, s, n$ : state function of the reduced model (18)

$f_{j}$ : function defined by (2) for $j=1, \ldots, n$ and corresponding

to the physical model, or function defined by (19) for $j=1$,

$r, j_{f}, s, n$ and corresponding to the reduced model.

$F$ : mole flow of the feed.

$\bar{F}:$ steady-state value of $F$.

$H_{j}$ : liquid holdup of tray $j$.

$H_{i}$ : liquid holdup of tray $j$ for the compartment of $m$ trays.

$\bar{H}=\sum_{1}^{m} \tilde{H}_{j}$.

$\bar{H}_{1}=H_{1}$

$\bar{H}_{r}=\sum_{2}^{j_{r}} H_{j}$ : the liquid holdup of the rectifying compartment.

$\bar{H}_{j_{F}}={ }^{i_{s}-1}{ }_{j}^{-1} H_{j}$ : the liquid holdup of the feed compartment.

* Received 18 February 1989; revised 8 March 1990; revised 3 August 1990; received in final form 4 September 1990. The original version of this paper was not presented at any IFAC meeting. This paper was recommended for publication in revised form by Associate Editor Y. Arkum under the direction of Editor H. Austin Spang III.

This work has been partially supported by ELF-FRANCE of the group ELF-AQUITAINE and E.E.C. under contract number EN3E/0134/F.

$\dagger$ École des Mines de Paris, Centre Automatique et Systèmes, 35 rue Saint Honore, 77305 Fontainebleau, France. Author to whom all correspondence should be addressed.

$\ddagger$ École des Mines de Paris, Centre Automatique et Systèmes, 60 Bd Saint Michel, 75006 Paris, France.
$\bar{H}_{s}=\sum_{j}^{n-1} H_{j}:$ the liquid holdup of the stripping compartment.

$\bar{H}_{n}=\stackrel{H}{s}_{n}$.

$j:$ tray index.

$j_{a}$ : index of the aggregation tray relative to the compartment of $m$ trays.

$i_{f}$ : index of the feed tray.

$j_{r}$ : defines the rectifying compartment $\left(2<j_{r}<j_{f}\right)$.

$j_{s}$ : defines the stripping compartment $\left(j_{f}<j_{s}<n-1\right)$.

$k$ : the equilibrium function.

$k\left(\bar{x}_{m+1}\right)$ : mole composition of the vapor entering the compartment of $m$ trays.

$k\left(x_{j}\right)$ : vapor mole fraction on tray $j$.

$L$ : reflux flow.

$\bar{L}$ : steady state value of $L$

$\tilde{L}:$ liquid flow in the compartment of $m$ trays.

$m$ : number of trays of the compartment.

$n$ : number of trays of the column.

$r$ : index of the aggregation tray relative to the rectifying compartment.

$s$ : index of the aggregation tray relative to the stripping compartment.

$t$ : the time.

$T_{j}=\Theta\left(x_{j}\right)$ : temperature of tray $j$ as a function of the liquid composition.

$v=\left(v_{1}, v_{2}\right)$ : the new control vector defined in Theorem 5

$V$ : reboiler vapor outflow.

$\bar{V}:$ steady state value of $V$

$\tilde{V}$ : vapor flow in the compartment of $\boldsymbol{m}$ trays

$x=\left(x_{i}\right)_{i=1, \ldots, n}$ : state vector of the physical model (1).

$x=\left(x_{j}\right)_{j=1, r, j, s, n}$ state vector of the reduced model (18).

$x^{s}:$ slow part of the state vector

$x^{F}$ : fast part of the state vector.

$x_{i}$ : liquid mole fraction on tray $j$ for $j=1, \ldots, n$ (a component of the physical state vector), or a component of the reduced state vector for $j=1, r, j_{f}, s, n$ [see (18)]

$\bar{x}_{j}:$ steady state value of $x_{j}$.

$\bar{x}_{0}$ : mole composition of the liquid entering the compartment of $m$ trays.

$X^{m}$ : function defined by Lemma 1 .

$y_{1}=x_{1}$ : the quality of the top product.

$y_{2}=x_{n}$ : the quality of the bottom product.

$\bar{y}_{1}$ : steady state value of $y_{1}$.

$\tilde{y}_{2}$ : steady state value of $y_{2}$.

$Y^{m}$ : function defined by Lemma 1 .

$z_{f}:$ feed composition.

$\bar{z}_{f}$ : steady state value of $z_{f}$.

$\varepsilon$ : small positive scalar.

$\Theta$ : real function that defines the tray temperature with respect to the tray liquid composition (bubble temperature). 
$\phi_{1}$ : function defined in Theorem 5 .

$\phi_{2}$ : function defined in Theorem 5 .

$r_{f}$ : relative to the feed.

$s$ : relative to slow dynamics.

$F$ relative to fast dynamics.

relative to the compartment of $m$ trays.

relative to the steady-state.

\section{INTRODUCTION}

COMPOSITION CONTROL of distillation columns has been studied extensively. The purpose is to maintain the product qualities at their setpoints, even if the feed flowrate and composition vary (generally, the feed composition is not measured). However, very few industrial columns maintain dual composition control. The main reason lies certainly in the difficulties attached to this problem [see for example Fuentes and Luyben (1983)]: strongly nonlinear and interactive system, very sluggish responses, deadtime in the composition measurements and large dimension.

In the literature relative to distillation dynamics and control, two generally separate streams can be identified: several papers emphasize modeling without strong influence on the control design, whereas several other papers concentrate on control without discussing the model.

In the "modeling stream", many physical models are proposed for simulation purposes [see Gallun and Holland (1982) for example]. Since the early study of Rosenbrock (1962), who established most of the qualitative results concerning such models, little theoretical progress has been observed. Other modeling ideas have been developed, for example by España and Landau (1978) and more recently by Benallou et al. (1986). They propose compartmental approximation techniques to obtain a simpler model of reduced order.

In the "control stream", many papers have been published and the field can be divided into many parts, in particular: linear predictive control [see for example Morari (1988) and Georgiou et al. (1988)]; adaptive control [see for example Agarwal and Seborg (1987)]; linear geometric control [see the pioneering work, using Wonham's (1974) approach, of Takamatsu et al. (1979) and more recently Kummel and Andersen (1987)]; and the nonlinear geometric approach [see Gauthier et al. (1983) and Lévine and Rouchon (1986)] applying the methods of Hirschorn (1981) and Isidori et al. (1981); see also the nonlinear control approach of Alsop and Edgar (1987) based on the approaches of Jakubczyk and Respondek (1980), Hunt et al.
(1983) and Krener (1984). Another related approach on extensive variable control can be found in Georgakis (1986).

To summarize, one can observe that firstly, distillation processes are carefully modeled and their dynamical properties are well established; secondly, recent developments of nonlinear control theory are able to provide efficient tools to incorporate the nonlinear aspect of this problem into the control design. In this perspective, our contribution is the following: firstly, we construct a simplified model where the nonlinear and qualitative properties of physical models are preserved (steady-state gains, molar fraction in $[0,1]$, global asymptotic stability, ... ); secondly, we compute a nonlinear control law, rejecting the feed composition perturbations, simple and robust enough to be implemented on a refinery depropanizer.

More precisely, we show that the classical distillation model studied by Rosenbrock (1962) can be approximated, via singular perturbation techniques [see Kokotovic (1984) for example], by a reduced-order model including only the slow transients but having the global asymptotic properties of the original model. This aggregated model proves very useful for control: we apply nonlinear perturbation rejection techniques [see Isidori (1989)] and obtain, around every slowly varying reference trajectory, a feedback law without singularity, producing asymptotically stable closed-loop dynamics, that can be synthesized via output feedback when temperature measurements are available. Though computed from a simplified model, this control law appears to be extremely robust when facing, in an industrial environment, delayed measurements and modeling errors. It is currently being used on a refinery depropanizer, providing, moreover, energy savings and increases in productivity and flexibility.

In the first section, a classical nonlinear physical model of binary distillation columns is recalled. The time-scale aggregation technique is presented and the qualitative properties of the resulting model are analyzed. In the second section, the existence of the control law, rejecting the feed composition perturbations on the aggregated model, and the closed-loop stability are proven. A robust control synthesis via output feedback is proposed and its implementation on a refinery depropanizer is presented. The last section is devoted to simulation comparisons between the obtained control law, the linear geometric one [Takamatsu et al. (1979)] and the nonlinear geometric one [inspired by Gauthier et al. (1983) and derived from the physical model of this paper]. 


\section{THE CONTROL MODEL}

After stating classical modeling assumptions, we recall the associated physical model which is reduced in Section 2.3 by time-scale considerations. For clarity's sake, the reduction is presented firstly on an arbitrary section of trays (compartment) and then extended to the overall column. In the whole section, the major tool for analyzing stability and convergence of our reduction method, is the global stability result of Rosenbrock (1962) that is recalled in Appendix A.

\subsection{Modeling assumptions}

A general description of distillation processes can be found in the book of Van Winkle (1967). The process studied is a classical binary distillation column [see for example España and Landau (1978)] as displayed in Fig. 1. The following assumptions are introduced:

1. On each tray, liquid and vapor phases are perfectly mixed and are at thermodynamic equilibrium.

2. The liquid molar holdup on each tray is constant. The pressure is constant and uniform. The vapor molar holdup on each tray is negligible.

3. The liquid molar inflow is equal to the liquid molar outflow on each tray. The vapor molar inflow is equal to the vapor molar outflow on

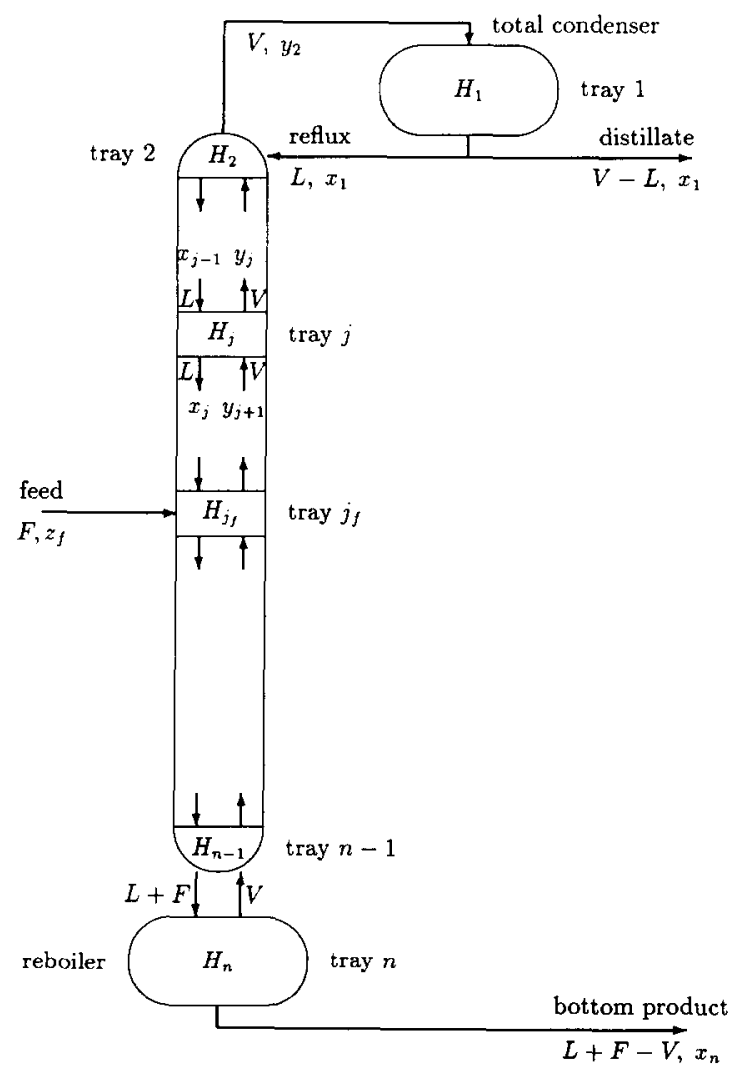

FIG. 1. Schematic diagram of a binary distillation column. each tray (with the exception of extreme trays 1 and $n$ ). The feed is a saturated liquid.

Assumption 1 means that the time constants of the mass transfer between liquid and vapor are much shorter than the resident time of each tray. Assumption 2 states that hydrodynamics, pressure and level dynamics are stable and fast enough to be neglected. This implies prefect level and pressure control. Generally, the tray's geometry, the pressure and level control loops are designed such that 1 and 2 are satisfied for smooth enough inputs $F, L$ and $V$, respectively the feed flow, the reflux flow and the reboiler vapor outflow. The last assumption, 3, is more restrictive and corresponds to the Lewis hypothesis [see Van Winkle (1967, p. 225)]. The control techniques which are used in this paper can be extended to more general models where energy balances are considered. Such an extension can be done only on a formal ground: the analysis of asymptotic stability of more complex models remains an open problem, even if simulations never display instabilities for reasonable operating conditions. Nevertheless, for several industrial columns-such as depropanizer, deethanizer, ...--assumption 3 provides a good approximation of the inner flow profiles.

Remark 1. For clarity's sake, uniform pressure and saturated liquid feed are considered. However, all the results of this paper remain valid if pressure drops corresponding to a given pressure profile and feed vapor fraction are introduced in the model: Rosenbrock's stability result applies and the closed-loop stability property (Theorem 5) can be extended to this case. It suffices to consider in place of the function $k$, introduced in Section 2.2, a collection of functions $k_{j}$ where $j$ denotes the tray index. $k_{j}$ enjoys the same properties as $k \mathrm{~s}$ but takes into account the pressure on tray $j$, $j=2, \ldots, n$. Also, denoting $v_{\text {frac }}$ the vapor fraction of the feed which is assumed to be measured or estimated, the liquid and vapor flows in the rectifying and stripping sections must be modified as follows: $L$ and $V$ must be replaced by $L$ and $V+v_{\text {frac }} F$ for the rectifying section; $L+F$ and $V$ must be replaced by $L+\left(1-v_{\text {frac }}\right) F$ and $V$ for the stripping section.

We now proceed as follows. Firstly, a physical model is obtained from assumptions 1,2 and 3 as in España and Landau (1978). For most industrial columns, this model is far too large for control purposes, but it can be used directly for simulation and comparisons. Secondly, this model is reduced by time-scale considerations to produce a satisfactory model for control. 


\subsection{A classical physical model}

Under assumptions 1, 2 and 3, the dynamic model of a binary column as displayed in Fig. 1 is derived from the balance equation on each tray for one component

$$
\left\{\begin{aligned}
& H_{1} \dot{x}_{1}=V k\left(x_{2}\right)-V x_{1} \\
& H_{j} \dot{x}_{j}= L x_{j-1}+V k\left(x_{j+1}\right)-L x_{j}-V k\left(x_{j}\right) \\
& j=2, \ldots, j_{f}-1 \\
& H_{j} \dot{x}_{j f}= L x_{j_{f}-1}+V k\left(x_{j_{f}+1}\right)-(L+F) x_{j f} \\
&-V k\left(x_{j f}\right)+F z_{f} \\
& H_{j} \dot{x}_{j}=(L+F) x_{j-1}+V k\left(x_{j+1}\right)-(L+F) x_{j} \\
&-V k\left(x_{j}\right), \quad j=j_{f}+1, \ldots, n-1 \\
& H_{n} \dot{x}_{n}=(L+F) x_{n-1}-(L+F-V) x_{n}-V k\left(x_{n}\right),
\end{aligned}\right.
$$

where:

$j$ denotes the tray index, $1 \leq j \leq n$;

$j=1$ corresponds to the reflux drum, $j=j_{f}$ to the feed tray and $j=n$ to the bottom $\left(3 \leq j_{f} \leq\right.$ $n-1)$;

$\left(H_{j}\right)_{(1 \leq j \leq n)}$ are the liquid holdups (constant); $x=\left(x_{j}\right)_{(1 \leq j \leq n)}$ are the liquid molar fractions;

$k\left(x_{j}\right)$ is the vapor molar fraction; $k$ corresponds to the thermodynamic equilibrium point of the binary mixture [see Prausnitz et al. (1980)]. We will call $k$ the equilibrium function;

$F$ and $z_{f}$ are the feed flow and composition, the perturbation variables; $F$ is measured whereas $z_{f}$ is not;

$L$ and $V$ are the reflux flow and the reboiler vapor outflow, the control variables.

System (1) is rewritten $\dot{x}=f\left(x, L, V, F, z_{f}\right)$ with

$$
f\left(x, L, V, F, z_{f}\right)=\left(f_{i}\left(x, L, V, F, z_{f}\right)\right)_{j=1, \ldots, n}
$$
where

$$
\left\{\begin{array}{c}
f_{1}\left(x, L, V, F, z_{f}\right)=\frac{V k\left(x_{2}\right)-V x_{1}}{H_{1}} \\
\text { for } j=2, \ldots, j_{f}-1: \\
f_{j}\left(x, L, V, F, z_{f}\right) \quad \frac{L x_{j-1}+V k\left(x_{j+1}\right)-L x_{i}-V k\left(x_{j}\right)}{H_{j}} \\
f_{j_{f}}\left(x, L, V, F, z_{f}\right) \\
=\frac{L x_{j_{f}-1}+V k\left(x_{j_{f}+1}\right)-(L+F) x_{j_{f}}-V k\left(x_{j_{f}}\right)-F z_{f}}{H_{j_{f}}} \\
\text { for } j=j_{f}+1, \ldots, n-1: \\
f_{j}\left(x, L, V, F, z_{f}\right) \\
=\frac{(L+F) x_{j-1}+V k\left(x_{j+1}\right)-(L+F) x_{j}-V k\left(x_{j}\right)}{H_{j}} \\
f_{n}\left(x, L, V, F, z_{f}\right) \\
=\frac{(L+F) x_{n-1}-(L+F-V) x_{n}-V k\left(x_{n}\right)}{H_{n}}
\end{array}\right.
$$

Notice that $f$ is linear with respect to $L, V, F$ and $F z_{f}$, and that $f$ is smooth if the equilibrium function $k$ is. We assume once and for all that:

Assumption 1. The inputs $L, V, F$ and $z_{f}$ are continuous time functions from $[0,+\infty[$ to $] 0,+\infty[$ such that for all $t \in[0,+\infty[, L(t)<V(t)<L(t)+$ $F(t)$ and $\left.z_{f}(t) \in\right] 0,1[$

Assumption 2. The equilibrium function $k$ and its derivative are continuous functions from $[0,1]$ to $[0,1], \frac{\mathrm{d} k}{\mathrm{~d} x}(x)>0$ for all $x \in[0,1], k(0)=0$ and $k(1)=1$ (see Fig. 2).

Remark 2. Assumption 1 means that the flow of the top and the bottom products is positive. Assumption 2 is satisfied for all binary systems: Malesinski (1965) derives from the second thermodynamic principle that the function $k$ is always an increasing function of $x$. For more general situations, see the analysis of Kwaalen $e t$ al. (1985). In practice, the equilibrium function $k$ is obtained by solving the algebraic nonlinear equations of the thermodynamic equilibrium. These equations depend on the particular choice of the thermodynamic model and are generally solved numerically. In the depropanizer application below the model of Soave (1972) is used.

The physical model considered is simpler than the model of Rosenbrock (1962) where vapor holdups and vapor Murphree efficiencies are considered whereas tray hydraulic effects are not taken into account. Our model can be seen as a particular case of Rosenbrock's, which contains twice the number of equations, by neglecting vapor holdups and assuming $100 \%$ tray efficiencies. For simplicity reasons, we demonstrate directly, for (1), the Rosenbrock open-loop results (and complete them with a spectrum property) by using the theorem of Appendix A.

Theorem 1. Assuming that Assumptions 1 and 2 hold, we have:

(i) For each initial condition $x^{0}$ in the closed

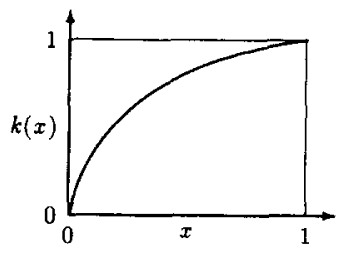

FIG. 2. The equilibrium function $k(x)$. 
subset $[0,1]^{n}$, the maximal solution of

$$
\dot{x}=f\left(x, L, V, F, z_{f}\right)
$$

is defined for every $t \in[0,+\infty[$ and satisfies $x(t) \in[0,1]^{n}$ for all $t \in[0,+\infty[$;

(ii) For each $L, V, F$ and $z_{f}$, there exists a unique steady-state $\bar{x}$ in $] 0,1{ }^{n}$, namely a unique solution of $f\left(\bar{x}, L, V, F, z_{f}\right)=0$; moreover, if $k$ satisfies $k(x)>x$ for all $x \in] 0,1[$, then $\bar{x}$ satisfies

$$
1>\bar{x}_{1}>\bar{x}_{2}>\cdots>\bar{x}_{n-1}>\bar{x}_{n}>0 \text {; }
$$

(iii) If $L, V, F$ and $z_{f}$ are constant and if $x^{0} \in[0,1]^{n}$, then the system is Lyapunov-stable [see Arnold (1974, p. 155)] and its solution converges to the unique steady state associated to $L, V, F$ and $z_{f}$; moreover, for every $x \in[0,1]^{n}$, the Jacobian matrix $\partial f / \partial x$ has real, distinct and negative eigenvalues.

Proof of (i). It is sufficient to prove that the vector field $f$ is oriented inwards on the boundary $\partial D$ of $D=[0,1]^{n}$. $\partial D$ is made of the points $\left(x_{1}, \ldots, x_{n}\right) \in[0,1]^{n}$ for which there exists $j \in\{1, \ldots, n\}$ such that $x_{j}=0$ or $x_{j}=1$. At such points, it suffices to prove that $\dot{x}_{j} \geq 0$ if $x_{i}=0$ and $\dot{x}_{i} \leq 0$ if $x_{j}=1$. This directly results from formula (2).

Proof of (ii) and (iii). We will prove simultaneously the existence and uniqueness of the steady-state, and the global asymptotic stability by applying Rosenbrock's theorem.

In our case, $p=n, \xi_{k}=H_{k} x_{k}$ for $k=$ $1, \ldots, n, \Omega=\prod_{k=1}^{n}\left[0, H_{k}\right]$ and system (1) is rewritten $\dot{\xi}=\phi(\xi)$ where $\phi$ is continuously differentiable. The dependence of $\phi$ with respect to $L, V, F$ and $z_{f}$ is omitted since they are assumed to be constant. The preceding proof of (i) implies that assertion (i) of Rosenbrock's theorem is satisfied. From (1) we see that for $i=2, \ldots, n-1, \quad \psi_{i}=0, \quad \psi_{1}=\frac{V-L}{H_{1}}$ and $\psi_{n}=$
$L+F-V$ $\frac{L+F-V}{H_{n}}$ with $\psi_{i}$ defined by (30). Assumption 1 implies that assertion (ii) of Rosenbrock's theorem is satisfied.

The Jacobian matrix $\partial \phi / \partial \xi$ is the matrix $J$ of Lemma 2 in Appendix A with $p=n$,

$$
\begin{aligned}
a=\left(\frac{L}{H_{1}}, \ldots, \frac{L}{H_{j_{f}-1}}, \frac{L+F}{H_{j_{f}}}, \ldots,\right. \\
\left.\qquad \frac{L+F}{H_{n-1}}, \frac{L+F-V}{H_{n}}\right)
\end{aligned}
$$

and

$$
b=\left(\frac{V-L}{H_{1}}, \frac{V}{H_{2}} \frac{\mathrm{d} k}{\mathrm{~d} x}\left(x_{2}\right), \ldots, \frac{V}{H_{n}} \frac{\mathrm{d} k}{\mathrm{~d} x}\left(x_{n}\right)\right) .
$$

Assumptions 1 and 2 imply that the vectors $a$ and $b$ have positive components. Consequently, assertion (iii) of Rosenbrock's theorem is satisfied. Moreover, Lemma 2 implies that the eigenvalues of $\partial \phi / \partial \xi$ are distinct, real and negative. Since $\phi(\xi)=H f\left(H^{-1} \xi, L, V, F, z_{f}\right)$ with $H=\operatorname{diag}\left[H_{1}, \ldots, H_{n}\right]$, the eigenvalues of $\partial f / \partial x$ have the same property. Assertion (iv) of Rosenbrock's theorem results from the tridiagonal structure of the system.

It remains necessary to prove $\bar{x} \in] 0,1{ }^{n}$ and the inequalities of (ii). We know that $\bar{x} \in[0,1]^{n}$ satisfies $f\left(\bar{x}, L, V, F, z_{f}\right)=0$. This is equivalent to

$$
\begin{gathered}
F z_{f}=(V-L) \bar{x}_{1}+(L+F-V) \bar{x}_{n} \\
k\left(\bar{x}_{j+1}\right)=\frac{L}{V} \bar{x}_{j}+\left(1-\frac{L}{V}\right) \bar{x}_{1}, \quad j=1, \ldots, j_{f}-1 \\
\bar{x}_{j-1}=\frac{V}{L+F} k\left(\bar{x}_{j}\right)+\left(1-\frac{V}{L+F}\right) \bar{x}_{n}, \\
j=j_{f}+1, \ldots, n,
\end{gathered}
$$

where (3) is obtained by summing all the equations of (1), (4) corresponds to the sum of the $j$ first equations and (5) to the sum of the $n-j+1$ last equations. If $\bar{x}_{1}=0$, then by induction on $j$ in (4) we have $\bar{x}_{j_{f}}=0$. Using (5) with $j=j_{f}+1$, we have $\bar{x}_{n}=0$. This is in contradiction with assumption 1 and (3). Similarly, we obtain that $\bar{x}_{1} \neq 1, \bar{x}_{n} \neq 0$ and $\bar{x}_{n} \neq 1$. Consequently $\left.\bar{x}_{1}, \bar{x}_{n} \in\right] 0,1[$. By induction on $j$ in (4) and (5), we have $\bar{x} \in] 0,1\left[{ }^{n}\right.$. If we suppose additionally that $k(x)>x$ for all $x \in] 0,1[$, then relations (4) and (5) give the desired inequalities.

\subsection{The reduced control model}

For industrial columns, the dimension of the above dynamic model is generally large (for a refinery depropanizer $n \approx 40$ ). It can be reduced by time-scale considerations. The standard form of a two-scale system is [see Kokotovic (1984) or Marino and Kokotovic (1988) for example]

$$
\left\{\begin{array}{l}
\dot{x}^{S}=f^{S}\left(x^{S}, x^{F}, u, w, \varepsilon\right) \\
\varepsilon \dot{x}^{F}=f^{F}\left(x^{S}, x^{F}, u, w, \varepsilon\right)
\end{array},\right.
$$

where $\left(x^{S}, x^{F}\right)$ is the state vector, the superscript $S$ (resp. $F$ ) standing for slow (resp. fast). $u$ is the control vector, $w$ the perturbation vector and $\varepsilon$ a small positive scalar. Under suitable assumptions, such a system can be reduced to its slow dynamics [by application of Tikhonov's theorem (Tikhonov et al., 1980) recalled in Appendix B)]

$$
\left\{\begin{array}{l}
\dot{x}^{S}=f^{S}\left(x^{S}, x^{F}, u, w, 0\right) \\
0=f^{F}\left(x^{S}, x^{F}, u, w, 0\right)
\end{array},\right.
$$

corresponding to $\varepsilon=0$. 
The model (1) is not in standard form (6). Nevertheless for physical reasons (the behavior of each tray is similar to that of any other, the resident time in one tray is much shorter than the resident in a "large" section of trays), we propose a choice of $\varepsilon$ and a diffeomorphic change of variables to express system (1) in standard form. More precisely, this can be done by splitting the column into a given number of sections of consecutive trays (called compartments), and by aggregation of each section separately. We now present the aggregation method on a given section of trays which gives an alternative model to the compartmental models of Benallou et al. (1986) or of España and Landau (1978).

2.3.1. The reduced model of a section of $m$ trays.

Preliminary results. Consider the section of $m$ trays displayed in Fig. 3. Its dynamic model is

$$
\left\{\begin{array}{c}
\tilde{H}_{1} \dot{\bar{x}}_{1}=\tilde{L} \tilde{x}_{0}+\tilde{V} k\left(\tilde{x}_{2}\right)-\tilde{L} \tilde{x}_{1}-\tilde{V} k\left(\tilde{x}_{1}\right) \\
\tilde{H}_{2} \dot{\bar{x}}_{2}=\tilde{L} \tilde{x}_{1}+\tilde{V} k\left(\tilde{x}_{3}\right)-\tilde{L} \tilde{x}_{2}-\tilde{V} k\left(\tilde{x}_{2}\right) \\
\vdots \\
\tilde{H}_{m-1} \dot{\tilde{x}}_{m-1}=\tilde{L} \tilde{x}_{m-2}+\tilde{V} k\left(\tilde{x}_{m}\right)-\tilde{L} \tilde{x}_{m-1} \\
\quad-\tilde{V} k\left(\tilde{x}_{m-1}\right) \\
\tilde{H}_{m} \dot{\hat{x}}_{m}=\tilde{L} \tilde{x}_{m-1}+\tilde{V} k\left(\tilde{x}_{m+1}\right)-\tilde{L} \tilde{x}_{m}-\tilde{V} k\left(\tilde{x}_{m}\right)
\end{array}\right.
$$

where:

$\left(\tilde{H}_{j}\right)_{(1 \leq j \leq m)}$ are the liquid holdups;

$\left(\tilde{x}_{j}\right)_{j=1, \ldots, m}$ are the liquid compositions;

$\tilde{L}$ and $V$ are the liquid and vapor flows entering the section;

$\tilde{x}_{0}$ and $k\left(\tilde{x}_{m+1}\right)$ are the compositions of the liquid and of the vapor entering the section ( $k$ is the previously defined equilibrium function).

The above system is denoted

$$
\dot{\bar{x}}=\tilde{f}\left(\tilde{x}, \tilde{L}, \tilde{V}, \tilde{x}_{0}, \tilde{x}_{m+1}\right),
$$

where $\tilde{x}=\left(\tilde{x}_{j}\right)_{j=1, \ldots, m}$ and $\tilde{f}$ denotes the right

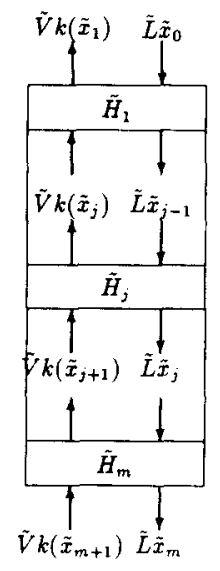

FIG. 3. A compartment of $m$ trays. hand side of (8). Similarly to the overall column, we have the following open-loop behavior.

Theorem 2. Assume assumption 2 and that the inputs $\tilde{L}, \tilde{V}, \tilde{x}_{0}$ and $\tilde{x}_{m+1}$ are continuous-time functions such that for all $t \in[0,+\infty[, \tilde{L}(t)$, $\tilde{V}(t) \in] 0,+\infty\left[, \quad \tilde{x}_{0}(t) \in[0,1]\right.$ and $\tilde{x}_{m+1}(t) \in[0,1]$. Then the following assertions hold true:

(i) For each initial condition $\tilde{x}^{0}$ in $[0,1]^{m}$, the maximal solution of (8) is defined on $[0,+\infty[$ and for all $t \in\left[0,+\infty\left[, \tilde{x}(t)\right.\right.$ remains in $[0,1]^{m}$;

(ii) For each $\tilde{L}, \tilde{V}, \tilde{x}_{0}$ and $\tilde{x}_{m+1}$ there exists a unique $\hat{x}=\left(\hat{x}_{j}\right)_{j=1, \ldots, m}$ in $[0,1]^{m}$ such that

$$
\tilde{f}\left(\hat{x}, \tilde{L}, \tilde{V}, \tilde{x}_{0}, \tilde{x}_{m+1}\right)=0 ;
$$

(iii) If the functions $\tilde{L}, \bar{V}, \tilde{x}_{0}$ and $\tilde{x}_{m+1}$ are constant and if the initial condition $\tilde{x}^{0}$ lies in $[0,1]^{m}$, then the system is Lyapunov-stable and

$$
\lim _{t \rightarrow+\infty} \tilde{x}(t)=\hat{x}
$$

where $\tilde{f}\left(\hat{x}, \tilde{L}, \tilde{V}, \tilde{x}_{0}, \tilde{x}_{m+1}\right)=0$ defines the steady-state $\hat{x}$.

The proof is a straightforward adaptation of the proof of Theorem 1 and left to the reader.

In the sequel we will use the following lemma relative to the steady-state $\hat{x}$ of (8).

Lemma 1. Assume assumption 2 and that $\tilde{L}>0, \tilde{V}>0$ and $0 \leq \tilde{x}_{0}, \tilde{x}_{m+1} \leq 1$. Consider the unique steady-state (Theorem 2) $\hat{x} \in[0,1]^{m}$ of (8). Then $k\left(\hat{x}_{1}\right)$ and $\hat{x}_{m}$ are continuously differentiable functions of $\tilde{L} / \tilde{V}, \quad \tilde{x}_{0}, \tilde{x}_{m+1}$ denoted respectively $Y^{m}\left(\tilde{L} / \tilde{V}, \tilde{x}_{0}, \tilde{x}_{m+1}\right)$ and $X^{m}\left(\tilde{L} / \bar{V}, \tilde{x}_{0}, \tilde{x}_{m+1}\right)$. They are related by the equation

$$
\begin{array}{r}
\tilde{L} X^{m}\left(\tilde{L} / \tilde{V}, \tilde{x}_{0}, \tilde{x}_{m+1}\right)+\tilde{V} Y^{m}\left(\tilde{L} / \tilde{V}, \tilde{x}_{0}, \tilde{x}_{m+1}\right) \\
=\tilde{L} \tilde{x}_{0}+\tilde{V} k\left(\tilde{x}_{m+1}\right)
\end{array}
$$

and satisfy

$$
\begin{array}{ll}
0 \leq Y^{m} \leq 1 & 0 \leq X^{m} \leq 1 \\
0<\frac{\partial Y^{m}}{\partial \tilde{x}_{0}}<\frac{\tilde{L}}{\tilde{V}} & 0<\frac{\partial X^{m}}{\partial \tilde{x}_{0}}<1 \\
0<\frac{\partial Y^{m}}{\partial \tilde{x}_{m+1}}<\frac{\mathrm{d} k}{\mathrm{~d} x}\left(\tilde{x}_{m+1}\right) & 0<\frac{\partial X^{m}}{\partial \tilde{x}_{m+1}} \\
& <\frac{\tilde{V} \mathrm{~d} k}{\tilde{L}} \frac{\mathrm{d} x}{\left(\bar{x}_{m+1}\right) .}
\end{array}
$$

Moreover, if $\hat{x}$ satisfies $\tilde{x}_{0}>\hat{x}_{1}>\cdots>\hat{x}_{r}>\tilde{x}_{m+1}$, then

$$
\frac{\partial Y^{m}}{\partial \tilde{L} / \tilde{V}}>0 \text { and } \quad \frac{\partial X^{m}}{\partial \tilde{L} / \tilde{V}}>0 .
$$


Proof. Notice that (9) results from the sum of all the equations of (8) at the steady state. It remains to prove (10) and (11). The proof is similar for $X^{m}$ and $Y^{m}$. Details are only given for $Y^{m}$. We will proceed by induction on $m$, the number of trays, to prove (10) and (11) for $Y^{m}$.

Let us prove that the result is true for $m=1$ and $Y^{1} . \hat{x}_{1}$ is given by (8):

$$
\frac{\tilde{L}}{\bar{V}} \tilde{x}_{1}+k\left(\tilde{x}_{1}\right)=\frac{\tilde{L}}{\bar{V}} \tilde{x}_{0}+k\left(\tilde{x}_{2}\right) ;
$$

since $k$ is a continously differentiable bijection from $[0,1]$ to $[0,1], \hat{x}_{1}$ exists, is unique and belongs to $[0,1] ; k\left(\hat{x}_{1}\right)$ is a regular function $Y^{1}$ of $\tilde{L} / \tilde{V}, \tilde{x}_{0}$ and $\tilde{x}_{2}$. The inequalities relative to $Y^{1}$ result from the derivation of the previous equation.

Assume that the result holds for $m-1 \geq 1$ and $Y^{m-1}$. Consider $\hat{x}=\left(\hat{x}_{j}\right)_{j=1, \ldots, m}$ the steady-state of $(8)$. Then $\left(\hat{x}_{2}, \ldots, \hat{x}_{m}\right)$ is the steady-state of the section made of trays 2 to $m$ corresponding to the $m-1$ last equation of (8). By the induction assumption, we have

$$
k\left(\hat{x}_{2}\right)=Y^{m-1}\left(\tilde{L} / \bar{V}, \hat{x}_{1}, \tilde{x}_{m+1}\right),
$$

where $Y^{m-1}$ is continuously differentiable and satisfies

$$
\begin{aligned}
& 0 \leq Y^{m-1} \leq 1 \\
& 0<\frac{\partial Y^{m-1}}{\partial \hat{x}_{1}}<\frac{\tilde{L}}{\tilde{V}} \\
& 0<\frac{\partial Y^{m-1}}{\partial \tilde{x}_{m+1}}<\frac{\mathrm{d} k}{\mathrm{~d} x}\left(\tilde{x}_{m+1}\right) .
\end{aligned}
$$

Moreover, if $\hat{x}_{1}>\hat{x}_{2}>\cdots>\hat{x}_{r}>\tilde{x}_{m+1}$, we have

$$
\frac{\partial Y^{m-1}}{\partial \tilde{L} / \tilde{V}}>0 .
$$

$\hat{x}_{1}$ is then given by

$\frac{\tilde{L}}{\bar{V}} \hat{x}_{1}+k\left(\hat{x}_{1}\right)-Y^{m-1}\left(\tilde{L} / \tilde{V}, \hat{x}_{1}, \tilde{x}_{m+1}\right)-\frac{\tilde{L}}{\bar{V}} \tilde{x}_{0}=0$,

the equation of (8) corresponding to the tray 1 . The left hand side of the above equation is an increasing continuously differentiable function of $\hat{x}_{1}$ (use inequality concerning the derivatives of $Y^{m-1}$ with respect to $\hat{x}_{1}$ ), nonpositive for $\hat{x}_{1}=0$ and non-negative for $\hat{x}_{1}=1$. Consequently, this function has a unique zero $\hat{x}_{1} \in[0,1] . k\left(\hat{x}_{1}\right)$ is a continuously differentiable function, $Y^{m}$, depending on $\tilde{L} / \tilde{V}, \tilde{x}_{0}$ and $\tilde{x}_{m+1}$ with values in $[0,1]$. The derivatives with respect to $\tilde{x}_{0}, \bar{x}_{m+1}$ and $\tilde{L} / \bar{V}$ of (12) immediately give the desired inequalities concerning the derivatives of $Y^{m}$.

Time-scale reduction. We suppose that the trays are comparable and that $1 \ll m$. Denote $\bar{H}=\sum_{1}^{m} \tilde{H}_{j}$ the section holdup. Consider the tray numbered $j_{a}, j_{a} \in\{1, \ldots, m\}$, called the aggregation tray. For each $j \neq j_{a}$, we set $\tilde{H}_{j}=\varepsilon \alpha_{j} \bar{H}$ with $0<\varepsilon \ll 1$ and $\alpha_{j} \approx 1$. We have $\tilde{H}_{j_{a}}=\bar{H}$ $\left(1-\sum_{j \neq j_{a}} \varepsilon \alpha_{j}\right)$. Consider the following change of state coordinate associated to $j_{a}$

$$
\left(\begin{array}{c}
\tilde{x}_{1} \\
\vdots \\
\tilde{x}_{j_{a}-1} \\
\tilde{x}_{j_{a}} \\
\tilde{x}_{j_{a}+1} \\
\vdots \\
\tilde{x}_{m}
\end{array}\right) \rightarrow\left(\begin{array}{c}
\tilde{x}_{1}^{F}=\tilde{x}_{1} \\
\vdots \\
\tilde{x}_{j_{a}-1}^{F}=\tilde{x}_{j_{a}-1} \\
\tilde{x}^{S}=\left(\sum_{1}^{m} \tilde{H}_{j} \tilde{x}_{j}\right) / \bar{H} \\
\tilde{x}_{j_{a}+1}^{F}=\tilde{x}_{j_{a}+1} \\
\vdots \\
\tilde{x}_{m}^{F}=\tilde{x}_{m}
\end{array}\right)
$$

where the composition of each tray $j \neq j_{a}$ remains unchanged and where the composition on the tray $j_{a}$ is replaced by the weighted sum of the compositions on the $m$ trays of the section. The state equations become

$$
\begin{aligned}
& \left(\begin{array}{c}
\varepsilon \alpha_{1} \bar{H} \dot{x}_{1}^{F}=\tilde{L} \bar{x}_{0}+\tilde{V} k\left(\bar{x}_{2}^{F}\right)-\tilde{L} \tilde{x}_{1}^{F}-\tilde{V} k\left(\tilde{x}_{1}^{F}\right) \\
\vdots \\
\varepsilon \alpha_{j_{a}-1} \bar{H}_{\dot{x}_{j_{a}-1}^{F}}=\tilde{L} \tilde{x}_{j_{a}-2}^{F}+\tilde{V} k\left(\frac{\tilde{x}^{s}-\varepsilon \sum_{j \neq j_{a}} \alpha_{j} \tilde{x}_{j}^{F}}{1-\varepsilon \sum_{j \neq j_{a}} \alpha_{j}}\right)
\end{array}\right. \\
& -\tilde{L} \bar{x}_{j_{a}-1}^{F}-\bar{V} k\left(\tilde{x}_{j_{a}-1}^{F}\right) \\
& \left\{\bar{H} \dot{\hat{x}}^{S}=\tilde{L} \tilde{x}_{0}+\tilde{V} k\left(\tilde{x}_{m+1}\right)-\tilde{L} \tilde{x}_{m}^{F}-\bar{V} k\left(\tilde{x}_{1}^{F}\right)\right. \\
& \varepsilon \alpha_{j_{a}+1} \bar{H} \dot{\bar{x}}_{j_{a}+1}^{F}=\tilde{L}\left(\frac{\tilde{x}^{S}-\varepsilon \sum_{j \neq j_{a}} \alpha_{j} \dot{x}_{j}^{F}}{1-\varepsilon \sum_{j \neq j_{a}} \alpha_{j}}\right) \\
& +\bar{V} k\left(\tilde{x}_{j_{a}+2}^{F}\right)-\tilde{L} \tilde{x}_{j_{a}+1}^{F}-\bar{V} k\left(\tilde{x}_{j_{a}+1}^{F}\right) \\
& \varepsilon \alpha_{m} \bar{H} \dot{\bar{x}}_{m}^{F}=\tilde{L} \bar{x}_{m-1}^{F}+\tilde{V} k\left(\tilde{x}_{m+1}\right)-\tilde{L} \bar{x}_{m}^{F}-\tilde{V} k\left(\tilde{x}_{m}^{F}\right)
\end{aligned}
$$

and are in the standard form (6). The slow and fast subsystems are thus described respectively by

$$
\left\{\begin{array}{c}
\bar{H} \dot{\bar{x}}^{S}=\tilde{L} \tilde{x}_{0}+\tilde{V} k\left(\tilde{x}_{m+1}\right)-\tilde{L} \tilde{x}_{m}^{F}-\tilde{V} k\left(\tilde{x}_{1}^{F}\right) \\
0=\tilde{L} \tilde{x}_{0}+\tilde{V} k\left(\tilde{x}_{2}^{F}\right)-\tilde{L} \tilde{x}_{1}^{F}-\tilde{V} k\left(\tilde{x}_{1}^{F}\right) \\
\vdots \\
0=\tilde{L} \tilde{x}_{j_{a}-2}^{F}+\tilde{V} k\left(\tilde{x}^{S}\right)-\tilde{L} \tilde{x}_{j_{a}-1}^{F}-\tilde{V} k\left(\tilde{x}_{j_{a}-1}^{F}\right) \\
0=\tilde{L} \tilde{x}^{S}+\tilde{V} k\left(\tilde{x}_{j_{a}+2}^{F}\right)-\tilde{L} \bar{x}_{j_{a}+1}^{F}-\tilde{V} k\left(\tilde{x}_{j_{a}+1}^{F}\right) \\
\vdots \\
0=\tilde{L} \tilde{x}_{m-1}^{F}+\tilde{V} k\left(\tilde{x}_{m+1}\right)-\tilde{L} \tilde{x}_{m}^{F}-\tilde{V} k\left(\tilde{x}_{m}^{F}\right)
\end{array}\right.
$$


and

$$
\left\{\begin{aligned}
\alpha_{1} \bar{H} \frac{\mathrm{d} \tilde{x}_{1}^{\mathrm{F}}}{\mathrm{d} \tau}= & \tilde{L} \tilde{x}_{0}+\tilde{V} k\left(\tilde{x}_{2}^{F}\right)-\tilde{L} \tilde{x}_{1}^{F}-\tilde{V} k\left(\tilde{x}_{1}^{F}\right) \\
\vdots & \\
\alpha_{j_{a}-1} \bar{H} \frac{\mathrm{d} \tilde{x}_{j_{a}-1}^{F}=}{\mathrm{d} \tau} & \tilde{L} \tilde{x}_{j_{a}-2}^{F}+\tilde{V} k\left(\tilde{x}^{S}\right) \\
& -\tilde{L}_{j_{j_{a}}-1}^{F}-\tilde{V} k\left(\tilde{x}_{j_{a}-1}^{F}\right) \\
\alpha_{j_{a}+1} \bar{H} \frac{\mathrm{d} \tilde{x}_{j_{a}+1}^{F}=}{\mathrm{d} \tau} \tilde{L} \tilde{x}^{S}+\tilde{V} k\left(\tilde{x}_{j_{a}+2}^{F}\right) & -\tilde{L} \tilde{x}_{j_{a}+1}^{F}-\tilde{V} k\left(\tilde{x}_{j_{a}+1}^{F}\right) \\
\vdots & \\
\alpha_{m} \overline{\mathrm{d}} \frac{\tilde{x}_{m}^{F}}{\mathrm{~d} \tau}= & \tilde{L} \tilde{x}_{m-1}^{F}+\tilde{V} k\left(\tilde{x}_{m+1}\right) \\
& -\tilde{L} \tilde{x}_{m}^{F}-\tilde{V} k\left(\tilde{x}_{m}^{F}\right)
\end{aligned}\right.
$$

with $\tau=t / \varepsilon$ in (16). We denote $\tilde{x}^{F}=\left(\tilde{x}_{j}^{F}\right)_{j \neq j_{a}}$.

Theorem 3. We assume that the equilibrium function satisfies Assumption 2 and that $\tilde{L}, \tilde{V}, \tilde{x}_{0}$ and $\tilde{x}_{m+1}$ are continuous time functions such that for all $t \in\left[0,+\infty[, \tilde{L}(t), \tilde{V}(t) \in] 0,+\infty\left[, \tilde{x}_{0}(t) \in\right.\right.$ $[0,1]$ and $\tilde{x}_{m+1}(t) \in[0,1]$ for all $t \geq 0$. If the initial conditions of $(14),\left(x^{S, 0}, x^{F, 0}\right)$, and of (15), $x^{s, 0}$, have their components in $[0,1]$, then (14) and (15) admit continuous solutions on $[0,+\infty[$, denoted respectively $\left(\tilde{x}^{S}(t, \varepsilon), \tilde{x}^{F}(t, \varepsilon)\right)$ and $\left(\tilde{x}_{0}^{S}(t), \tilde{x}_{0}^{F}(t)\right)$, satisfying

$$
\lim _{\varepsilon \rightarrow 0^{+}}\left(\begin{array}{l}
\tilde{x}^{S}(t, \varepsilon) \\
\tilde{x}^{F}(t, \varepsilon)
\end{array}\right)=\left(\begin{array}{l}
\bar{x}_{0}^{S}(t) \\
\tilde{x}_{0}^{F}(t)
\end{array}\right),
$$

uniformly on every interval of the form $[\alpha, T]$ with $0<\alpha<T$.

Proof. Let us verify that all the assertions of the Tikhonov's theorem (Tikhonov et al., 1980) (see Appendix B) are satisfied:

- Existence of the solution of the perturbed system (14): Theorem 2 proves that (14) admits a solution $\left(\tilde{x}^{S}(t, \varepsilon), \tilde{x}^{F}(t, \varepsilon)\right)$ on $[0,+\infty[$;

- Existence and stability of the fast subsystem (16): in (16) $\tilde{L}, \tilde{V}, \tilde{x}_{0}, \bar{x}_{m+1}$ and $\tilde{x}^{S}$ are constant parameters; consequently, (16) is made of two decoupled section of trays, the first one corresponding to the trays 1 to $j_{a}-1$, the second one to the trays $j_{a}+1$ to $m$; Theorem 2 implies that if $\tilde{x}^{S} \in[0,1]$, system (16) admits a unique steady state, and if the initial condition lies in $[0,1]^{m-1}$, then $(16)$ is globally asymptotically stable; moreover, its Jacobian matrix has distinct, real and negative eigenvalues;

- Existence of the solution of the slow subsystem (15): if $\tilde{x}_{0}^{S} \in[0,1]$, the algebraic equations of (15) has a unique solution, $\tilde{x}_{0}^{F}$, corresponding to the steady state of (16); using Lemma 1, (15) becomes

$$
\begin{aligned}
\tilde{H} \dot{\bar{x}}_{0}^{S}= & \tilde{L} \tilde{x}_{0}+\tilde{V} k\left(\tilde{x}_{m+1}\right)-\tilde{V} Y^{j_{a}-1}\left(\tilde{L} / \tilde{V}, \tilde{x}_{0}, \bar{x}_{0}^{S}\right) \\
& -\tilde{L} X^{n-j_{a}}\left(\tilde{L} / \bar{V}, \tilde{x}_{0}^{S}, \tilde{x}_{m+1}\right) ;
\end{aligned}
$$

the proof of the existence of the solution for $t \in[0,+\infty$ [ and remaining in $[0,1]$ is similar to the proof of (i) in Theorem 1 [use (10) of Lemma 1] and is left to the reader.

Remark 3. Expressed in a less mathematical form, the result of Theorem 3 becomes very simple. The dynamics of the whole section can be approximated by the dynamics of a section where the holdup profile is modified as follows: the trays $j \neq j_{a}$ have no holdup $\left(0 \rightarrow \tilde{H}_{j}\right)$, the tray $j_{a}$ has the section holdup $\left(\sum_{1}^{m} \tilde{H}_{j} \rightarrow \tilde{H}_{j_{a}}\right)$. Notice that the global holdup remains unchanged.

2.3.2. The reduced model of the column. Consider now the overall column. The choice of the compartments (sections of consecutive trays) has to take into account several considerations concerning the holdups. For most columns, the holdup profile is as follows:

- The holdups on external trays 1 and $n$ (reflux drum and bottom) are much more important than the holdups on any other tray (trays $2, \ldots, n-1)$

- The holdups on external trays are comparable to the global holdup of all other trays;

- The holdups on trays 2 to $n-1$ are comparable.

The aggregated model should simultaneously have a small dimension and represent correctly the column dynamics.

We can consider that the two external trays have their own slow dynamics. For the trays 2 to $n-1$, the number of compartments constitutes a degree of freedom. In the case of the depropanizer described in the discussion below, open-loop trajectory comparisons between the physical model and different aggregated models (aggregated models of orders 3, 4 and 5, corresponding to aggregations of trays 2 to $n-1$ in respectively 1,2 and 3 compartments), are displayed in Fig. 4. They correspond to variations of the feed composition slightly more severe than what is usually observed in practice. They show that a good tradeoff between accuracy and dimension can be obtained with an aggregated model of order 5 .

In the sequel, we consider a 5-compartment aggregated model as displayed in Fig. 5:

- The two external trays remain unchanged;

- The other trays are decomposed into 3 similar 

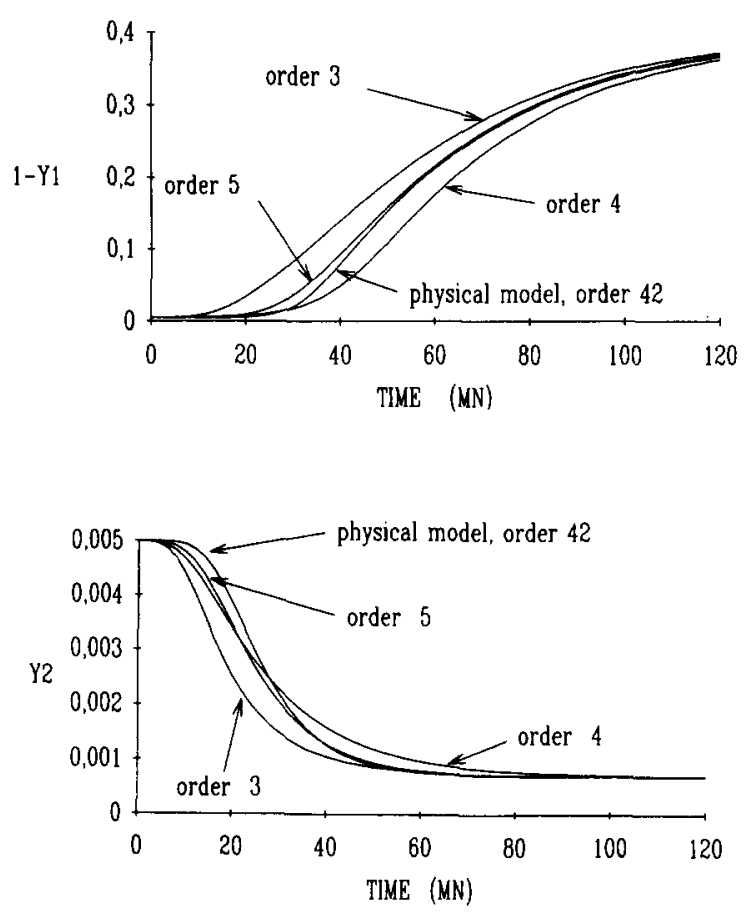

FIG. 4. Simulated open-loop responses to a step change in the feed composition corresponding to several aggregated models of a depropanizer.

sections: the rectifying section (trays 2 to $j_{r}$ ) with its aggregation tray $r$; the feed section (trays $j_{r}+1$ to $j_{s}-1$ ) with its aggregation tray $j_{f}$; the stripping section (trays $j_{s}$ to $n-1$ ) with

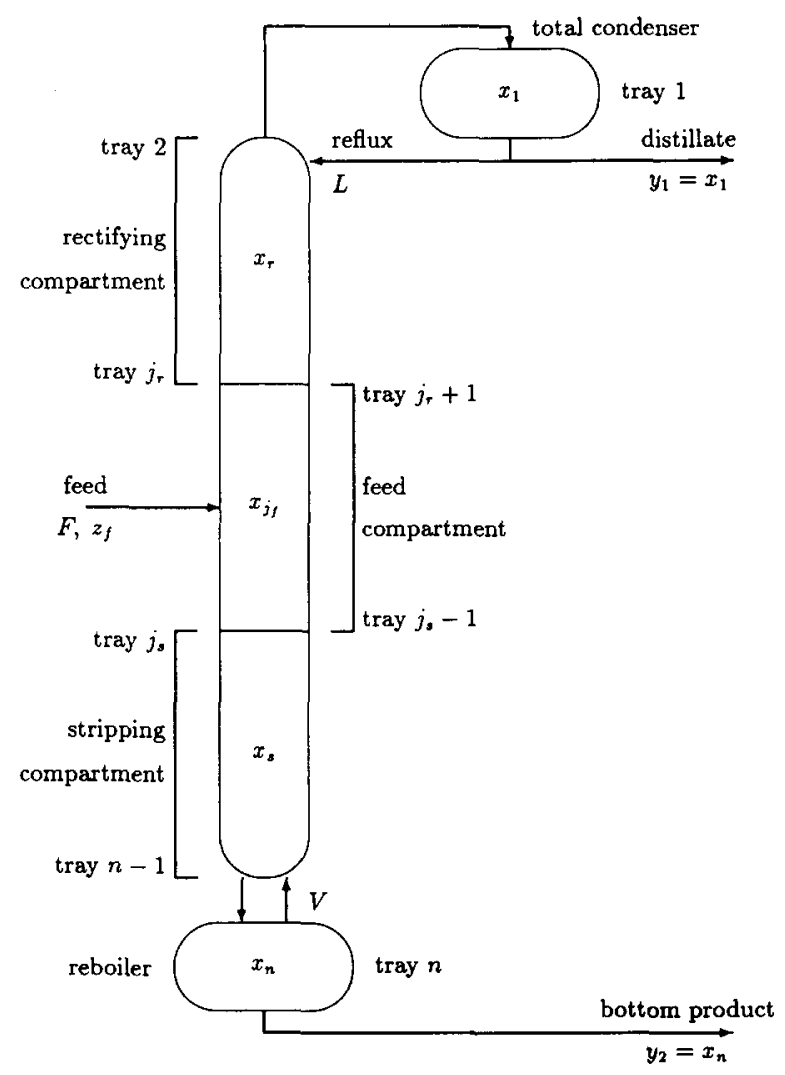

FIG. 5. The aggregation in 5 compartments. its aggregation tray $s\left(2<r<j_{r}<j_{f}<j_{s}<s<\right.$ n).

According to Theorem 3 (see also Remark 3), the reduced model is described by the differential-algebraic system

$$
\left\{\begin{array}{c}
\bar{H}_{1} \dot{x}_{1}=V k\left(x_{2}\right)-V x_{1} \\
0=L x_{j-1}+V k\left(x_{j+1}\right)-L x_{j}-V k\left(x_{j}\right) \\
j=2, \ldots, r-1 \\
\bar{H}_{r} \dot{x}_{r}=L x_{r-1}+V k\left(x_{r+1}\right)-L x_{r}-V k\left(x_{r}\right) \\
0=L x_{j-1}+V k\left(x_{j+1}\right)-L x_{j}-V k\left(x_{j}\right) \\
j=r+1, \ldots, j_{f}-1 \\
\bar{H}_{j_{f}} \dot{x}_{j_{f}}=L x_{j_{f}-1}+V k\left(x_{j_{f}+1}\right)-(L+F) x_{j_{f}} \\
\quad-V k\left(x_{j f}\right)-F z_{f} \\
0=(L+F) x_{j-1}+V k\left(x_{j+1}\right)-(L+F) x_{j} \\
-V k\left(x_{j}\right), j=j_{f}+1, \ldots, s-1 \\
\bar{H}_{s} \dot{x}_{s}=(L+F) x_{s-1}+V k\left(x_{s+1}\right)-(L+F) x_{s} \\
\quad-V k\left(x_{s}\right) \\
0=(L+F) x_{j-1}+V k\left(x_{j+1}\right)-(L+F) x_{j} \\
-V k\left(x_{j}\right), \quad j=s+1, \ldots, n-1 \\
\bar{H}_{n} \dot{x}_{n}=(L+F) x_{n-1}-(L+F-V) x_{n}-V k\left(x_{n}\right)
\end{array}\right.
$$

where

$$
\begin{aligned}
& \bar{H}_{1}=H_{1}, \quad \bar{H}_{r}=\sum_{2}^{j_{r}} H_{j}, \quad \bar{H}_{i_{f}}=\sum_{j_{r}+1}^{j_{s}-1} H_{j}, \\
& \bar{H}_{s}=\sum_{j_{s}}^{n-1} H_{j}, \quad \bar{H}_{n}=H_{n} .
\end{aligned}
$$

The substitution of the algebraic equations into the 5 differential equations preserves the tridiagonal structure of the original system (1) and gives the aggregated model

$$
\left\{\begin{array}{l}
\dot{x}_{1}=f_{1}\left(x_{1}, x_{r}, L, V\right) \\
\dot{x}_{r}=f_{r}\left(x_{1}, x_{r}, x_{j_{f}}, L, V\right) \\
\dot{x}_{j_{f}}=f_{j_{f}}\left(x_{r}, x_{j_{f}}, x_{s}, L, V, F, z_{f}\right) \\
\dot{x}_{s}=f_{s}\left(x_{j_{f}}, x_{s}, x_{n}, L, V, F\right) \\
\dot{x}_{n}=f_{n}\left(x_{s}, x_{n}, L, V, F\right) \\
y_{1}=x_{1} \\
y_{2}=x_{n} .
\end{array}\right.
$$

It results that $x^{S}=\left(x_{1}, x_{r}, x_{i f}, x_{s}, x_{n}\right)$, and $x^{F}$ corresponds to the liquid compositions of the remaining trays. (18) is called the control model and:

$y_{1}$ and $y_{2}$ are the outputs;

$L$ and $V$ are the control variables;

$z_{f}$ is the perturbation;

$F$ is a measured input;

The vector field $f$ can be defined only with the 
functions $Y^{m}$ of Lemma 1 ,

$$
\left\{\begin{array}{l}
\bar{H}_{1} f_{1}\left(x_{1}, x_{r}, L, V\right) \\
\quad=-(V-L) x_{1}+V Y^{r-2}\left(L / V, x_{1}, x_{r}\right)-L x_{1} \\
\bar{H}_{r} f_{r}\left(x_{1}, x_{r}, x_{j_{f}}, L, V\right) \\
\quad=L x_{1}-V Y^{r-2}\left(L / V, x_{1}, x_{r}\right) \\
\quad+V Y^{j_{f}-r-1}\left(L / V, x_{r}, x_{j_{f}}\right)-L x_{r} \\
\bar{H}_{j_{f}} f_{j_{f}}\left(x_{r}, x_{j_{f}}, x_{s}, L, V, F, z_{f}\right) \\
\quad=L x_{r}-V Y^{j_{f}-r-1}\left(L / V, x_{r}, x_{j_{f}}\right) \\
\quad+V Y^{s-j_{f}-1}\left((L+F) / V, x_{j_{f}}, x_{s}\right) \\
\quad-(L+F) x_{j_{s}} \\
\bar{H}_{s} f_{s}\left(x_{j_{f}}, x_{s}, x_{n}, L, V, F\right)=(L+F) x_{j_{f}} \\
\quad-V Y^{s-j_{f}-1}\left((L+F) / V, x_{j_{f}}, x_{s}\right) \\
\quad+V Y^{n-s-1}\left((L+F) / V, x_{s}, x_{n}\right) \\
\quad-(L+F) x_{s} \\
\bar{H}_{n} f_{n}\left(x_{s}, x_{n}, L, V, F\right)=(L+F) x_{s} \\
\quad-V Y^{n-s-1}\left((L+F) / V, x_{s}, x_{n}\right) \\
\quad-(L+F-V) x_{n} .
\end{array}\right.
$$

From now on, we shall only work with this control model. For obvious notational reasons, $x$ and $f$, previously used for the physical model, remain unchanged since no ambiguity is possible: $x=x^{S}=\left(x_{1}, x_{r}, x_{j}, x_{s}, x_{n}\right)$ and $f=$ $\left(f_{1}, f_{r}, f_{i f}, f_{s}, f_{n}\right)$.

The proposed reduction preserves the openloop behavior of the physical model.

Theorem 4. Assume that Assumptions 1 and 2 hold. Then we have the following assertions:

(i) For each initial condition $x^{0}$ in the open subset $[0,1]^{5}$, the maximal solution of $(18)$ is defined on $\left[0,+\infty\left[\right.\right.$ and remains in $[0,1]^{5}$.

(ii) For each $L, V, F$ and $z_{f}$, there exists a unique steady state $\bar{x}$ in $] 0,1\left[{ }^{5}\right.$ of system (18). Moreover, if $k(x)>x$ for all $x \in] 0,1[$ then $\bar{x}$ satisfies

$$
1>\bar{x}_{1}>\bar{x}_{r}>\bar{x}_{j_{f}}>\bar{x}_{s}>\bar{x}_{n}>0 .
$$

(iii) If the functions $L, V, F$ and $z_{f}$ are constant and if $x^{0}$ lies in $[0,1]^{5}$, then $(18)$ is Lyapunov-stable and its solution converges to the unique steady-state associated to $L, V, F$ and $z_{f}$; moreover, for every $x \in[0,1]^{5}$, the Jacobian matrix $\partial f / \partial x$ has real, distinct and negative eigenvalues.

The proof is a straightforward adaptation of that of Theorem 1 using Lemma 1.

Remark 4. Our method is of the same spirit as Benallou et al. (1986) or as España and Landau (1978) since it produces a reduced model of the column where dynamics of trays are replaced by dynamics of sections of trays.

However, if we assume piecewise constant equilibrium, our model is not a special case of Benallou et al. (1986) compartmental model. Let us recall the Benallou et al. compartmentation assumption: "The dynamic behavior of a section of stages, or a compartment, can be represented by that of a single stage having the same holdup as the total compartment holdup and the composition of the compartment sensitive stage". The dynamic behavior of our aggregation tray does not satisfy this assumption since, even if steady state coincide with the ones of the compartment sensitive stage, transients may differ. More precisely, if we apply the reduction technique of Benallou et al. (1986) to the 5 compartments case as displayed on Fig. 5, we obtain a reduced model whose structure does not remain tridiagonal whereas our aggregated model does. This results from the application of the equations (24) to (32) in Benallou et al. (1986). One can verify that the differential equation corresponding to the feed compartment depends, in Benallou et al. (1986), on $x_{1}$ and $x_{n}$, the product compositions, whereas, in our aggregated model (18) it does not. On the other hand, as in Benallou et al. (1986), the steady states of the outputs $y_{1}=x_{1}$ and $y_{2}=x_{n}$ are preserved whatever the inputs $L, V, F$ and $z_{f}$ are.

Contrary to España and Landau (1978), we do not use bilinear approximations of (1) for which the calculated molar fractions do not necessarily remain in $[0,1]$. Moreover, no identification of compartmental parameters is needed here.

Note also that the qualitative dynamic behavior of our aggregated model is deduced from the stability analysis of Rosenbrock (1962) whereas, for the reduced models of Benallou et al. (1986) and España and Landau (1978), this analysis remains to be done.

Remark 5. We can enrich our model by including hydraulic effects due to the liquid flowing down from tray to tray. These dynamics that have been neglected in the physical model (1), are, for most industrial columns, much faster that the slowest part of the dynamics of the compositions and include time constants similar to those of the fast part of the composition dynamics. Moreover, the application of the Tikhonov theorem to a physical model including such hydraulic effects will produce the same aggregated model (18) since for the slow hydraulic model the liquid holdups remain constant. This is why we presented the analysis without such hydraulic effects. To fix 

the Routh-Hurwitz criterion [see Gantmacher (1966) for example] on the linear tangent approximation at the steady state.

According to (i), the unique static feedback is locally given by

$$
\begin{aligned}
& L=\Xi_{1}\left(x_{1}, x_{r}, x_{s}, x_{n}, F, \phi_{1}\left(x_{1}, v_{1}\right), \phi_{2}\left(x_{n}, v_{2}\right)\right) \\
& V=\Xi_{2}\left(x_{1}, x_{r}, x_{s}, x_{n}, F, \phi_{1}\left(x_{1}, v_{1}\right), \phi_{2}\left(x_{n}, v_{2}\right)\right),
\end{aligned}
$$

where $\Xi_{1}$ and $\Xi_{2}$ are continuously differentiable functions. The closed-loop dynamics are thus

$$
\left\{\begin{aligned}
\dot{x}_{1}= & \phi_{1}\left(x_{1}, v_{1}\right) \\
\dot{x}_{r}= & f_{r}\left(x_{1}, x_{r}, x_{j_{f}},\right. \\
& \Xi_{1}\left(x_{1}, x_{r}, x_{s}, x_{n}, F, \phi_{1}\left(x_{1}, v_{1}\right), \phi_{2}\left(x_{n}, v_{2}\right)\right), \\
& \left.\Xi_{2}\left(x_{1}, x_{r}, x_{s}, x_{n}, F, \phi_{1}\left(x_{1}, v_{1}\right), \phi_{2}\left(x_{n}, v_{2}\right)\right)\right) \\
\dot{x}_{i_{f}}= & f_{j_{f}}\left(x_{r}, x_{j r}, x_{s}, \Xi_{1}\left(x_{1}, x_{r}, x_{s}, x_{n}, F,\right.\right. \\
& \left.\phi_{1}\left(x_{1}, v_{1}\right), \phi_{2}\left(x_{n}, v_{2}\right)\right), \\
& \Xi_{2}\left(x_{1}, x_{r}, x_{s}, x_{n}, F,\right. \\
& \left.\left.\phi_{1}\left(x_{1}, v_{1}\right), \phi_{2}\left(x_{n}, v_{2}\right)\right), F, z_{f}\right) \\
\dot{x}_{s}= & f_{s}\left(x_{j r}, x_{s}, x_{n}, \Xi_{1}\left(x_{1}, x_{r}, x_{s}, x_{n}, F, \phi_{1}\left(x_{1}, v_{1}\right),\right.\right. \\
& \left.\phi_{2}\left(x_{n}, v_{2}\right)\right), \Xi_{2}\left(x_{1}, x_{r}, x_{s}, x_{n}, F, \phi_{1}\left(x_{1}, v_{1}\right),\right. \\
& \left.\left.\phi_{2}\left(x_{n}, v_{2}\right)\right), F\right) \\
\dot{x}_{n}= & \phi_{2}\left(x_{n}, v_{2}\right) .
\end{aligned}\right.
$$

The dynamics of $x_{1}$ and $x_{n}$ are decoupled and stable by assumption. Consequently, the stability, around the steady-state $\bar{x}$, is ensured if the zero dynamics, obtained by setting $\phi_{1}$ to 0 , $\phi_{2}$ to $0, x_{1}$ to $\bar{y}_{1}$ and $x_{n}$ to $\bar{y}_{2}$ in $(23)$, is asymptotically stable. That is, if

$$
\left\{\begin{aligned}
& \dot{x}_{r}= f_{r}\left(\bar{y}_{1}, x_{r}, x_{j f}, \Xi_{1}\left(\bar{y}_{1}, x_{r}, x_{s}, \bar{y}_{2}, F, 0,0\right),\right. \\
&\left.\Xi_{2}\left(\bar{y}_{1}, x_{r}, x_{s}, \bar{y}_{2}, F, 0,0\right)\right) \\
& \dot{x}_{j_{f}}= f_{j_{f}}\left(x_{r}, x_{i f}, x_{s}, \Xi_{1}\left(\bar{y}_{1}, x_{r}, x_{s}, \bar{y}_{2}, F, 0,0\right),\right. \\
&\left.\Xi_{2}\left(\bar{y}_{1}, x_{r}, x_{s}, \bar{y}_{2}, F, 0,0\right), F, z_{f}\right) \\
& \dot{x}_{s}= f_{s}\left(x_{j_{f}}, x_{s}, \bar{y}_{2}, \Xi_{1}\left(\bar{y}_{1}, x_{r}, x_{s}, \bar{y}_{2}, F, 0,0\right)\right. \\
&\left.\Xi_{2}\left(\bar{y}_{1}, x_{r}, x_{s}, \bar{y}_{2}, F, 0,0\right), F\right)
\end{aligned}\right.
$$

is asymptotically stable.

In the remaining part of this proof, all functions are evaluated at the steady-state. For simplicity's sake, we also denote $\frac{\partial L}{\partial x_{r}}$ in place of $\frac{\partial \Xi_{1}}{\partial x_{r}}$, and the same for $V$. The equations (22) defining the control law become

$$
\left\{\begin{array}{l}
Y^{r-2}\left(\frac{L}{V}, \bar{y}_{1}, x_{r}\right)-\bar{y}_{1}=0 \\
\frac{L+F}{V} x_{s}-\left(\frac{L+F}{V}-1\right) \bar{y}_{2} \\
\quad-Y^{n-s-1}\left(\frac{L+F}{V}, x_{s}, \bar{y}_{2}\right)=0
\end{array}\right.
$$

when $\phi_{1}=\phi_{2}=0$. The inequalities (11) imply that

$$
\begin{gathered}
\frac{V}{L+F} \frac{\partial L}{\partial x_{r}}=\frac{\partial V}{\partial x_{r}}<0, \quad \frac{\partial L / V}{\partial x_{r}}<0, \\
\frac{\partial(L+F) / V}{\partial x_{r}}=0,
\end{gathered}
$$

$$
\frac{\partial L / V}{\partial x_{s}}=0, \quad \frac{\partial L}{\partial x_{s}}=\frac{L}{V} \frac{\partial V}{\partial x_{s}}>0, \quad \frac{\partial(L+F) / V}{\partial x_{s}}<0 .
$$

Using (19), the closed-loop system (24) can be rewritten as follows:

$$
\begin{aligned}
\bar{H}_{r} \dot{x}_{r} & =-g_{1}+g_{r} \\
\bar{H}_{j_{f}} \dot{x}_{j_{f}} & =F z_{f}-g_{r}-g_{s} \\
\bar{H}_{s} \dot{x}_{s} & =-g_{n}+g_{s},
\end{aligned}
$$

where $g_{1}, g_{r}, g_{s}$ and $g_{n}$ are the following functions of $\left(x_{r}, x_{j}, x_{s}\right)$

$$
\begin{aligned}
g_{1}\left(x_{r}, x_{s}\right)= & \left(V\left(x_{r}, x_{s}\right)-L\left(x_{r}, x_{s}\right)\right) \bar{y}_{1} \\
g_{r}\left(x_{r}, x_{j_{f}}, x_{s}\right)= & V\left(x_{r}, x_{s}\right) Y^{j_{f}-r-1}\left(\frac{L}{V}\left(x_{r}\right), x_{r}, x_{j_{r}}\right) \\
& -L\left(x_{r}, x_{s}\right) x_{r} \\
g_{s}\left(x_{r}, x_{j_{f}}, x_{s}\right)= & \left(L\left(x_{r}, x_{s}\right)+F\right) x_{j_{f}} \\
& -V\left(x_{r}, x_{s}\right) Y^{s-j_{f}-1}\left(\frac{L+F}{V}\left(x_{s}\right), x_{j_{f}}, x_{s}\right) \\
g_{n}\left(x_{r}, x_{s}\right)= & \left(L\left(x_{r}, x_{s}\right)+F-V\left(x_{r}, x_{s}\right)\right) \bar{y}_{2} .
\end{aligned}
$$

Let us now compute the tangent linearization to (27). (26) and $g_{r}-g_{1}=0$ at the steady-state imply that $\frac{\partial}{\partial x_{s}}\left(g_{r}-g_{1}\right)=0$. Symmetrically, we have $\frac{\partial}{\partial x_{r}}\left(g_{s}-g_{n}\right)=0$. Consequently the matrix $A$ corresponding to the tangent model of the zero dynamics (27) has the form

$$
A=\left(\begin{array}{ccc}
\frac{a_{1}+a_{2}}{\bar{H}_{r}} & \frac{a_{3}}{\bar{H}_{r}} & 0 \\
-\frac{a_{2}+b_{2}}{\bar{H}_{j_{f}}} & -\frac{a_{3}+b_{3}}{\bar{H}_{j_{f}}} & -\frac{a_{4}+b_{4}}{\bar{H}_{j_{f}}} \\
0 & \frac{b_{3}}{\bar{H}_{s}} & \frac{b_{1}+b_{4}}{\bar{H}_{s}}
\end{array}\right),
$$

where:

$$
\begin{array}{llll}
a_{1}=-\frac{\partial g_{1}}{\partial x_{r}}, & a_{2}=\frac{\partial g_{r}}{\partial x_{r}}, & a_{3}=\frac{\partial g_{r}}{\partial x_{j r}}, & a_{4}=\frac{\partial g_{r}}{\partial x_{s}}, \\
b_{1}=-\frac{\partial g_{n}}{\partial x_{s}}, & b_{2}=\frac{\partial g_{s}}{\partial x_{r}}, & b_{3}=\frac{\partial g_{s}}{\partial x_{j r}}, & b_{4}=\frac{\partial g_{s}}{\partial x_{s}} .
\end{array}
$$


The eigenvalues $\lambda$ of $A$ are solution of

$$
\left|\begin{array}{ccc}
\frac{a_{1}+a_{2}}{\bar{H}_{r}}-\lambda & \frac{a_{3}}{\bar{H}_{r}} & 0 \\
-\frac{a_{2}+b_{2}}{\bar{H}_{j r}} & -\frac{a_{3}+b_{3}}{\bar{H}_{i_{f}}}-\lambda & -\frac{a_{4}+b_{4}}{\bar{H}_{j_{f}}} \\
0 & \frac{b_{3}}{\bar{H}_{s}} & \frac{b_{1}+b_{4}}{\bar{H}_{s}}-\lambda
\end{array}\right|=0,
$$

or equivalently of

$$
\left|\begin{array}{ccc}
a_{1}+a_{2}-\bar{H}_{r} \lambda & a_{3} & 0 \\
a_{1}-b_{2}-\bar{H}_{r} \lambda & -\bar{H}_{i_{f}} \lambda & b_{1}-a_{4}-\bar{H}_{s} \lambda \\
0 & b_{3} & b_{1}+b_{4}-\bar{H}_{s} \lambda
\end{array}\right|=0
$$

We have

$$
\begin{aligned}
& a_{1}+a_{2} \\
& =\frac{\partial(L-V)}{\partial x_{r}} \bar{y}_{1}+\frac{\partial\left(V Y^{i_{r}-r-1}-L x_{r}\right)}{\partial x_{r}} \\
& =\frac{L+F-V}{L+F} \bar{y}_{1} \frac{\partial L}{\partial x_{r}}+\frac{V Y^{j_{r}-r-1}-(L+F) x_{r}}{L+F} \frac{\partial L}{\partial x_{r}} \\
& +V\left(\frac{\partial Y^{j_{f}-r-1}}{\partial L / V}\right) \frac{\partial L / V}{\partial x_{r}}+V \frac{\partial Y^{j_{f}-r-1}}{\partial x_{r}}-L \\
& =\frac{F\left(\bar{y}_{1}-x_{r}\right)}{\underbrace{L+F}_{>0}} \underbrace{\frac{\partial L}{\partial x_{V}}}_{<0}+V \underbrace{\left(\frac{\partial Y^{i_{f}-r-1}}{\partial L / V}\right)}_{>0} \underbrace{\frac{\partial L / V}{\partial x_{r}}}_{<0} \\
& +\underbrace{V \frac{\partial Y^{j_{f}-r-1}}{\partial x_{r}}-L}_{<0},
\end{aligned}
$$

(use $g_{1}=g_{r}$ at the steady-state, (25), (10) with $m=j_{f}-r-1$ and $x_{r}$ playing the role of $\tilde{x}_{0}$, $\left.\bar{y}_{1}>\bar{x}_{r}\right)$. Consequently $a_{1}+a_{2}<0$. Similar computations give

$$
\begin{gathered}
a_{3}>0, \quad a_{1}-b_{2}<0, \quad b_{1}-a_{4}<0, \\
b_{3}>0, \quad b_{1}+b_{4}<0 .
\end{gathered}
$$

Denote

$$
\alpha_{1}=-\frac{a_{1}+a_{2}}{\bar{H}_{r}}, \quad \alpha_{2}=-\frac{a_{1}-b_{2}}{\bar{H}_{r}}, \quad \alpha_{3}=\frac{a_{3}}{\bar{H}_{j_{f}}},
$$

and

$$
\beta_{1}=-\frac{b_{1}+b_{4}}{\bar{H}_{s}}, \quad \beta_{2}=-\frac{b_{1}-a_{4}}{\bar{H}_{s}}, \quad \beta_{3}=\frac{b_{3}}{\bar{H}_{j_{f}}} .
$$

$\alpha_{1}, \alpha_{2}, \alpha_{3}, \beta_{1}, \beta_{2}$ and $\beta_{3}$ are positive. The characteristic polynomial of $A$ is then

$$
\begin{aligned}
\lambda^{3} & +\left(\alpha_{1}+\beta_{1}+\alpha_{3}+\beta_{3}\right) \lambda^{2} \\
& +\left(\alpha_{1} \beta_{1}+\alpha_{3} \alpha_{2}\right. \\
& \left.+\alpha_{3} \beta_{1}+\beta_{3} \beta_{2}+\beta_{3} \alpha_{1}\right) \lambda \\
& +\alpha_{2} \alpha_{3} \beta_{1}+\beta_{2} \beta_{3} \alpha_{1} .
\end{aligned}
$$

We have

$$
\begin{aligned}
& \alpha_{1}+\beta_{1}+\alpha_{3}+\beta_{3}>0 \\
& \alpha_{1} \beta_{1}+\alpha_{3} \alpha_{2}+\alpha_{3} \beta_{1}+\beta_{3} \beta_{2}+\beta_{3} \alpha_{1}>0
\end{aligned}
$$

$$
\begin{aligned}
& \alpha_{2} \alpha_{3} \beta_{1}+\beta_{2} \beta_{3} \alpha_{1}>0 \\
& \left(\alpha_{1}+\beta_{1}+\alpha_{3}+\beta_{3}\right)\left(\alpha_{1} \beta_{1}+\alpha_{3} \alpha_{2}+\alpha_{3} \beta_{1}+\beta_{3} \beta_{2}\right. \\
& \left.\quad+\beta_{3} \alpha_{1}\right)>\alpha_{2} \alpha_{3} \beta_{1}+\beta_{2} \beta_{3} \alpha_{1} .
\end{aligned}
$$

The desired stability result follows from the Routh-Hurwitz criterion.

Remark 7 . The preceding result is only a local stability result. Simulations show that stronger stability properties should be expected, but we have no proof of them. On the other hand, it should be noticed that, in (i) of Theorem 5, $\phi_{1}$ and $\phi_{2}$ can be chosen in order to follow an arbitrary slow reference model. It results that set-point changes are easily handled by such a control technique. An integral action can be added through $v_{1}$ and $v_{2}$ in order to remove static offsets between the control model and the real column.

Remark 8. Equation (20) shows that $x_{j,}$ is not required to compute the control law. This results from the special tridiagonal structure of the reduced model (18) and from the disturbance rejection method. More precisely, one can show, using the results of Isidori (1989), that the characteristic numbers of the outputs $y_{1}$ and $y_{2}$ with respect to the control are zero which means that the control variables affect the first time derivatives of $y_{1}$ and $y_{2}$. The perturbation rejection method consists in making unobservable all the state variables which are affected by the perturbations and which need a larger number of derivations to affect $y_{1}$ and $y_{2}$. Indeed, otherwise they would reintroduce the effects of the perturbations in the outputs. This explains why the feedback makes $x_{r}, x_{j,}$ and $x_{s}$ unobservable through $y_{1}$ and $y_{2}$ and why $x_{j}$, which affects the outputs after 2 time derivations, does not appear in the control law. Note that the same results would hold true with more compartments and that the feedback scheme would remain the same. Namely, this would produce only an increase of the number of unobservable state variables by a feedback law still depending on 4 state variables.

Remark 9. The closed-loop analysis has been done under the condition that $k(x)>x$ for all $x \in$ ]0, 1[. Azeotropic mixtures [see Prausnitz et al. (1980) for example] do not satisfy such conditions. Nevertheless, the same result can in fact be proven by assuming that $k(x)>x$, $\forall x \in] 0, a[$ and $k(x)<x, \quad \forall x \in] a, 1[$ where $a \in] 0,1[$ is the azeotropic composition $(k(a)=$ $a)$. The reason is that the steady-state compositions are all on the same side of $a$ if $z_{f} \neq a$.

\subsection{Synthesis by output feedback}

The control law of Theorem 5 depends on $\left(x_{1}, x_{r}, x_{s}, x_{n}, F\right)$ and on the reference model 
(21). In practice, the product compositions $\left(x_{1}, x_{n}\right)$ and the feed flow $F$ are measured. But the average compositions $\left(x_{r}, x_{s}\right)$ in the rectifying and stripping compartments are not measured. Nevertheless, $\left(x_{r}, x_{s}\right)$ can be estimated by means of well placed temperatures which leads to a reasonable approximation of the control law.

With the equilibrium function $k$, the equations of the thermodynamic equilibrium also give the temperature $T$ on each tray as a function of its liquid composition $x$ (see Prausnitz et al., 1980): $T=\Theta(x)$. We now prove that $T_{r}$ and $T_{s}$, the temperatures on the aggregation trays $r$ and $s$, can be considered, at the order zero in $\varepsilon$, as functions of $x_{r}$ and $x_{s}$ respectively, and thus can be seen as additional outputs for system (18).

Let us return to the compartment of $m$ trays of 2.3.1. Consider the temperature of the aggregated tray $j_{a}, \tilde{T}_{j_{a}}$, and its liquid composition, $\tilde{x}_{j_{a}}$. We have: $\tilde{T}_{j_{a}}=\Theta\left(\tilde{x}_{j_{a}}\right)$. $\tilde{x}_{j_{a}}$ depends on the slow variable $\tilde{x}^{S}$ but also on the fast variables $\tilde{x}^{F}$ as follows:

$$
\tilde{x}_{j_{a}}=\frac{\tilde{x}^{S}-\varepsilon \sum_{j \neq j_{a}} \alpha_{j} \tilde{x}_{j}^{F}}{1-\varepsilon \sum_{j \neq j_{a}} \alpha_{j}} .
$$

At the order 0 in $\varepsilon, \tilde{x}_{j_{a}}=\tilde{x}^{S}$. Consequently, $\tilde{T}_{j_{a}}=\Theta\left(\tilde{x}^{S}\right)+O(\varepsilon)$. For the overall column, we have similarly

$$
T_{r}=\Theta\left(x_{r}\right)+O(\varepsilon) \text { and } T_{s}=\Theta\left(x_{s}\right)+O(\varepsilon) .
$$

This implies that $x_{r}$ and $x_{s}$ can be estimated via temperatures on aggregation trays $r$ and $s$. Moreover, these estimates do not contain fast components at the order 0 in $\varepsilon$. Thus the feedback law where $x_{r}$ and $x_{s}$ are replaced by $\Theta^{-1}\left(T_{r}\right)$ and $\Theta^{-1}\left(T_{s}\right)$ neither destabilizes the neglected fast dynamics nor the aggregated closed-loop system. Otherwise stated, the proposed synthesis by output feedback does not remix the time scales of the original system (for an extended discussion see Kokotovic (1984) or Marino and Kokotovic (1988)].

To summarize, the control law can be computed with the following online measurements:

- The product compositions, $x_{1}$ and $x_{n}$;

- The rectifying temperature on tray $r, T_{r}$;

- The stripping temperature on tray $s, T_{s}$;

- The feed flow, $F$.

We have observed that the position of the aggregation trays $r$ and $s$ does not require great precision. For the depropanizer described below, several choices of $r$ and $s$ have been explored. The corresponding simulations show only slight differences. An important byproduct of this approach is that, when the setpoints remain unchanged for a long period, the measurements of the product composition $\left(1-y_{1}, y_{2}\right)$ are not necessary. Consequently, failures on these measurements can appear without significantly affecting the behavior of the control law.

Notice that it results from Remark 8 that the number of on-line measurements (temperatures) does not depend on the number of compartments of the reduced model.

\subsection{Implementation on a refinery depropanizer}

The control law of Theorem 5 has been implemented on a refinery depropanizer (a binary column splitting a mixture of propane and butane into two products: the top product, essentially propane, and the bottom product, essentially butane). This depropanizer has the following characteristics:

- 42 theoretical trays $(n=42)$, feed on tray 21 $\left(j_{f}=21\right)$.

- The holdup profile is as follows: on tray 1 (reflux drum): $60 \mathrm{kmol}$; on trays 2 to 41 : $2 \mathrm{kmol}$; on tray 42 (bottom): $30 \mathrm{kmol}$.

- The top pressure: 15 bar.

- A typical steady-state is: saturated liquid feed flow of $5 \mathrm{kmol} / \mathrm{min}$ with a propane molar fraction around 0.35 , a reflux flow of $5 \mathrm{kmol} / \mathrm{min}$, a reboiler vapor outflow of $7 \mathrm{kmol} / \mathrm{min}$, product purities of $0.5 \%$ butane in the top product, and of $0.5 \%$ propane in the bottom product.

For this column, the modeling assumptions are valid, and the hypothesis of Theorems 1, 3, 4 and 5 are satisfied. The real-time control law depends on:

- The molar fraction of butane in the top product;

- The molar fraction of propane in the bottom product;

- The rectifying temperature on tray 11 ;

- The stripping temperature on tray 33;

- The feed flowrate;

- The top pressure as a parameter in the thermodynamic calculations.

It should be mentioned that the two composition measurements are obtained with a delay greater than $5 \mathrm{~min}$. The first control $L$, the reflux flow, is directly measured and regulated. The second control $V$, the reboiler vapor outflow, is proportional to the reboiler duty which is measured and regulated. The thermodynamic model (the functions $k$ and $\Theta$ ), used to represent the binary mixture propane-butane, is borrowed from Soave (1972).

We now present records of real data relative 
to this depropanizer. On Fig. 6, the variations over 10 hours of the product compositions $\left(1-y_{1}\right.$ and $y_{2}$ ), of the control variables ( $L$ and $V)$ and of the measured input $(F)$, are displayed. At time 0 , the control law is switched on; the objectives are set to $0.5 \%$ butane in the distillate (setpoint of $1-y_{1}$ ) and to $0.3 \%$ propane in the bottom product (setpoint of $y_{2}$ ). These data suggest two comments. Firstly, though important initial offsets exist between outputs and setpoints, the objectives are reached in 5 hours (the time-constants of the linear first-order reference models are around 2 hours). Secondly, the outputs are only slightly modified in spite of severe variations of the feed fiow $F$ (more than $40 \%$ in $15 \mathrm{~min}$ ). This demonstrates that the nonliner control law works in a large range of operating conditions and rejects the perturbations asymptotically.

To conclude this section, we have compared our control technique with the following classical SISO method:

- The reflux flow $L$ is proportional to the feed flow $F$ with a gain slowly adapted by PI controller depending on the top composition $y_{1}$

- The reboiler duty proportional to $V$ is controlled through PI action depending on the stripping temperature $T_{s}$.

Figure 7 displays on-line data relative to the same depropanizer where the nonlinear control is removed after $t=360 \mathrm{~min}$ and is replaced, in the absence of feed perturbations, by the SISO control described above. The bottom quality remains acceptable whereas the top quality
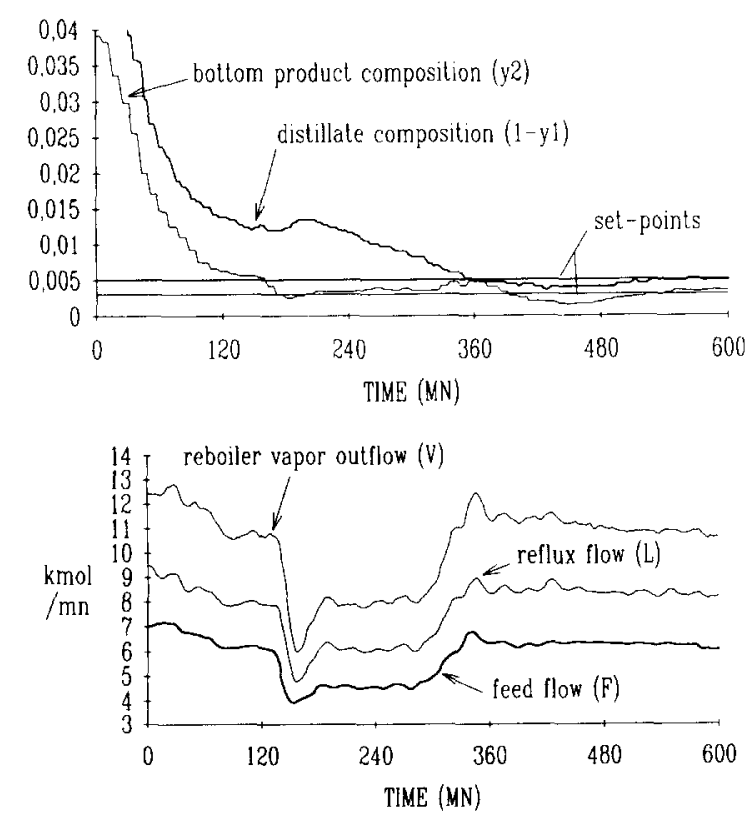

FIG. 6. Plant data; implementation of the aggregated nonlinear control on a refinery depropanizer.

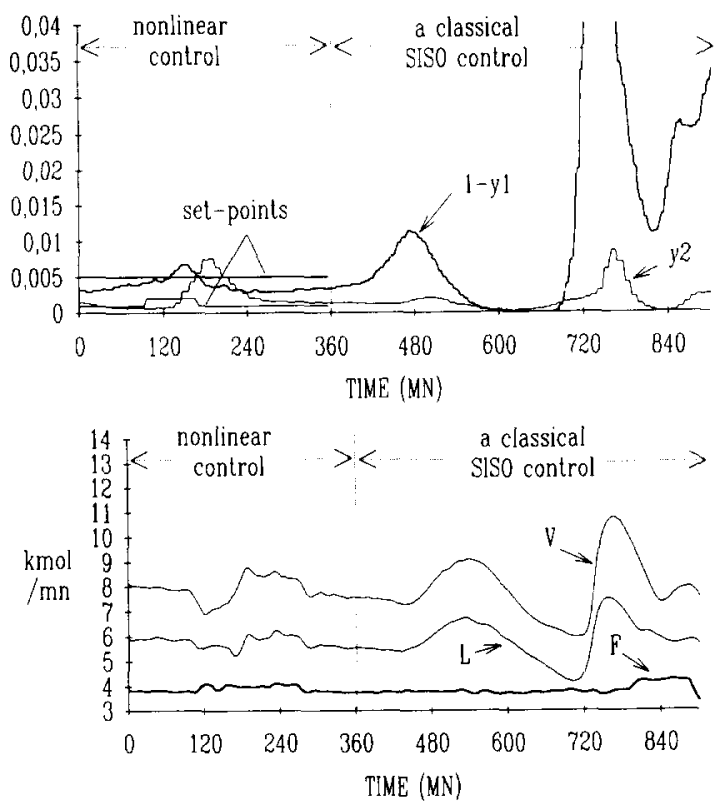

FIG. 7. Plant data; comparison between the aggregated nonlinear control and a classical SISO control technique on a refinery depropanizer.

slowly becomes out of specifications. This SISO controls the quality of one of the two products in a quite satisfactory way, but it is unable to control simultaneously the top and bottom product qualities.

\section{DISCUSSION}

All the dynamic simulations presented in this section correspond to the refinery depropanizer of the preceding section and are obtained via the dynamic simulator SPEEDUP [User Manual (1988)]. Numerical integrations start from the same steady-state characterized by:

- Feed flowrate $5 \mathrm{kmol} / \mathrm{min}$, reflux flowrate $5.088 \mathrm{kmol} / \mathrm{min}$, vapor leaving the reboiler $6.957 \mathrm{kmol} / \mathrm{min}$;

- Column pressure 15 bar;

- Feed composition: $2.5 \%$ ethane, $35 \%$ propane, $60 \% n$-butane, $2.5 \% n$-pentane.

During the first $10 \mathrm{~min}$, the feed compositions change to the new values: $2.5 \%$ ethane (unchanged), $20 \%$ propane, $75 \%$-butane, $2.5 \% n$-pentane (unchanged). After that, all the entries remain unchanged. The thermodynamic model used to represent the liquid-vapor equilibria is the Soave model (1972). The open-loop responses can be seen on Fig. 4 where a similar perturbation of the feed composition is introduced.

The set-points are: $0.5 \%$-butane in the top product, $0.5 \%$ propane in the bottom product. Notice that apart from the two key components (propane, $n$-butane), we add two other secondary ones, present in practice in small quantities.

In the control law of Theorem 5, the output 
dynamics (the functions $\phi_{1}$ and $\phi_{2}$ ) are arbitrary stable dynamics. For the simulation tests, we choose

$$
\phi_{1}\left(y_{1}, v_{1}\right)=\frac{v_{1}-y_{1}}{\alpha}, \quad \phi_{2}\left(y_{2}, v_{2}\right)=\frac{v_{2}-y_{2}}{\alpha},
$$

where $\alpha=10 \mathrm{~min}$ is constant, $v_{1}=0.995$ is the top set-point and $v_{2}=0.005$ the bottom one (the component chosen to write the balance equations is the $n$-butane).

Our control technique is now compared with other linear and nonlinear nonaggregated methods. We do not consider errors on the state measurements since an observer ought to be designed in each case and the comparisons would be hazardous. Only robustness versus delays is studied.

\subsection{Why nonlinear control?}

We compare, by simulation, the performance of the nonlinear control law of Theorem 5 with the linear geometric control law of Takamatsu $e t$ al. (1979). This linear control law is the solution of the linear system

$$
\begin{aligned}
a_{1,2} b_{2,1} \delta L+a_{1,2} b_{2,2} \delta V= & \ddot{\delta} x_{1}-\left(a_{1,1}^{2}+a_{1,2} a_{2,1}\right) \\
& \times \delta x_{1}-a_{1,2}\left(a_{1,1}+a_{2,2}\right) \\
& \times \delta x_{2}-a_{1,2} a_{2,3} \delta x_{3} \\
b_{n, 1} \delta L+b_{n, 2} \delta V=\dot{\delta} x_{n}- & a_{n, n-1} \delta x_{n-1} \\
& -a_{n, n} \delta x_{n}-b_{n, 1} \delta F,
\end{aligned}
$$

where

$\left(a_{i, j}\right)_{1 \leq i, j \leq n}$ and $\left(b_{i, j}\right)_{1 \leq i \leq n, j=1,2}$ are obtained by linearization at the steady-state;

$\left(\delta x_{j}\right)_{1 \leq j \leq n}$ are the deviations of the state $x$ (see system (1)), $(\delta L, \delta V)$ the deviations of the control $(L, V)$ and $\delta F$ the deviation of the measured input $F$;

$\ddot{\delta} x_{1}$ and $\delta x_{n}$ are the closed-loop output dynamics, chosen linear and stable:

$$
\left\{\begin{array}{l}
\ddot{\delta} x_{1}=-\left(\frac{1}{\theta}+\frac{1}{2 \theta}\right) \dot{\delta} x_{1}-\frac{1}{2 \theta^{2}} \delta x_{1} \\
\dot{\delta} x_{n}=-\frac{\delta x_{n}}{\theta}
\end{array}\right.
$$

with $\theta=5 \mathrm{~min}, \quad$ a constant and $\dot{\delta} x_{1}=$ $a_{1,1} \delta x_{1}+a_{1,2} \delta x_{2}$.

In simulations, we suppose that the part of the state required for control is measured directly (the composition of propane on tray 1 and 2, used for the control, are calculated by 1 minus the true compositions of $n$-butane on these trays).

Figure 8 corresponds to the closed-loop output responses to feed composition perturbations, firstly when the state is perfectly known and secondly when a measurement delay of $5 \mathrm{~min}$ is
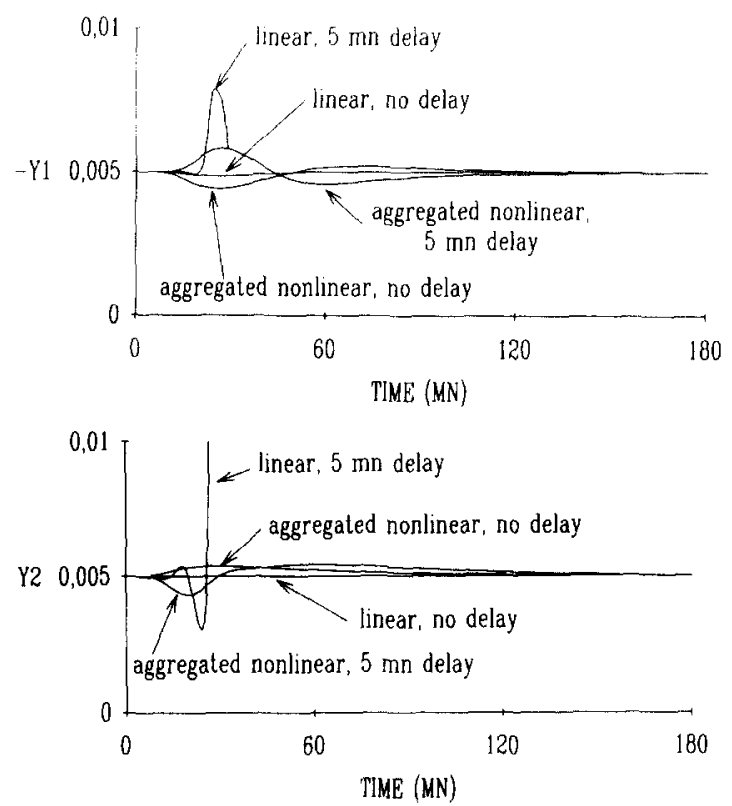

FIG. 8. Simulation data; comparison between the aggregated nonlinear control law and the linear geometric control law (depropanizer, step change of the feed composition).

introduced. This delay corresponds to the resident time in the chromatograph, the composition sensor generally used for a depropanizer. The linear control law works better than the nonlinear aggregated one if the measurements are perfect and without delays. But, the nonlinear aggregated control law is less sensitive to measurement delays, whereas the linear one blows up in their presence.

In other simulations, we have observed that the parameter $\theta$ of (28) must be carefully chosen: if $\theta$ is too large, for instance greater than $10 \mathrm{~min}$, the linear control law destabilizes the column. The gains of the control law must be large enough to maintain the linear model in its validity region that seems to be small.

\subsection{Why aggregation?}

We have also compared the nonlinear aggregated control law with the nonlinear control law rejecting feed composition disturbances in the system (1).

We have proven in Lévine and Rouchon (1986) that, at the steady-state, the nonlinear control law of Gauthier et al. (1983) is singular. In order to bypass this difficulty, one can look for nonmaximal invariant distributions (see Isidori, 1989). Since on the singularity $x_{1}=$ $k\left(x_{2}\right)$, the reader can verify that this leads to rejecting the perturbation on the new output functions $\left(y_{1}=k\left(x_{2}\right), y_{2}=x_{n}\right)$. That is, we change the top output $\left(x_{1}\right)$ to the propane composition of the vapor leaving tray $2\left(k\left(x_{2}\right)\right)$. Clearly, since $H_{1} \dot{x}_{1}=V\left(k\left(x_{2}\right)-x_{1}\right)$, if $x_{2}$ is constant then $x_{1}$ is also constant. With these new outputs the control law is regular near the 

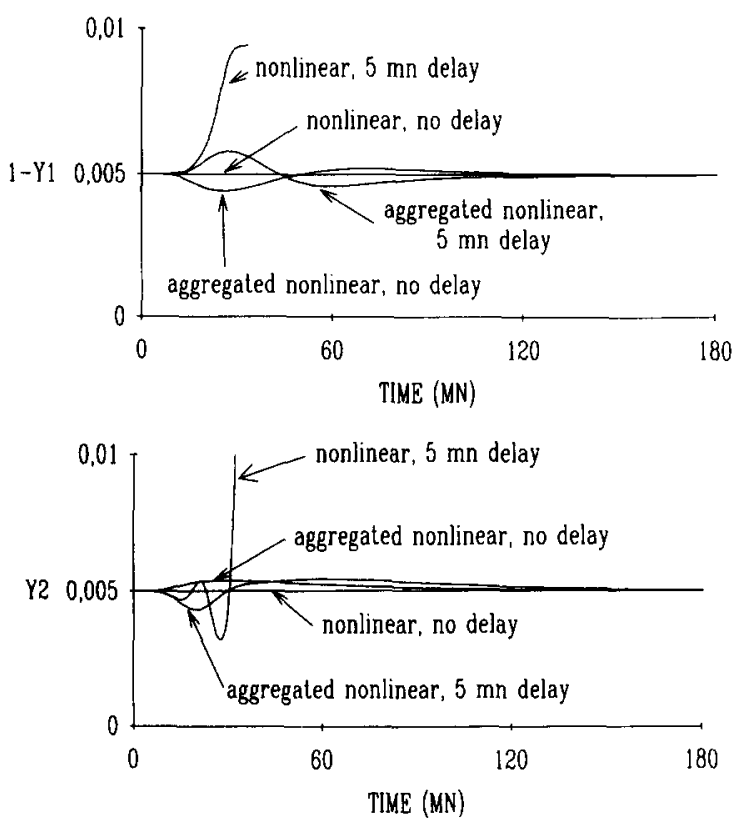

Fig. 9. Simulation data; comparison between the aggregated nonlinear control law and the complete nonlinear one elaborated on the physical model (depropanizer, step change of the feed composition).

steady-state and given by

$$
\left\{\begin{array}{l}
\frac{\mathrm{d} k}{\mathrm{~d} x}\left(x_{2}\right) \frac{x_{1}-x_{2}}{H_{2}} L+\frac{\mathrm{d} k}{\mathrm{~d} x}\left(x_{2}\right) \frac{k\left(x_{3}\right)-k\left(x_{2}\right)}{H_{2}} V \\
=\frac{v_{1}-k\left(x_{2}\right)}{\beta} \\
\frac{x_{n-1}-x_{n}}{H_{n}} L+\frac{x_{n}-k\left(x_{n}\right)}{H_{n}} V \\
=\frac{v_{2}-x_{n}}{\beta}+\frac{x_{n}-x_{n-1}}{H_{n}} F,
\end{array}\right.
$$

where we chose linear stable output dynamics with $\beta=10 \min \left(v_{1}=0.995\right.$ and $\left.v_{2}=0.005\right)$.

As for the linear geometric control, two simulations have been made: the first one with perfect state measurements and no delay, the second one with a measurement delay of $5 \mathrm{mn}$ (see Fig. 9). The complete nonlinear control law works perfectly without delays. But the linear geometric control destabilizes the column in the presence of delays. Instead of the linear geometric control, the choice of the parameter $\beta$ in (29) is not important: with perfect state knowledge, stability is ensured if $\beta>0$.

\section{CONCLUSIONS}

We have applied the nonlinear perturbation rejection techniques to an aggregated model of distillation columns. The obtained control law is shown to be robust and simple to implement. It is actually working on two depropanizers and two debutanizers of ELF-FRANCE.

Another by-product of our control technique concerns the design of the instrumentation of the columns. The proposed control law uses intermediate temperatures; their positions can be adjusted in order to give the best closed-loop responses (composition and noise sensitivities).

The same technique can be extended to other counter-current separation processes, as in Duchêne (1988), where counter-current mixersettler extractors are studied and similar results are obtained. Moreover, the extensions of these results to more complex distillation columns (more than two components, several feeds, side products, networks of columns) seems to be reasonable.

The implementation on the reflnery depropanizer has shown that controlling product qualities has several industrial interests, in particular:

- Energy savings: the purity margins that the operator maintains with the final product specification can be reduced, as well as the associated energy consumption (for the depropanizer, savings are greater than $15 \%$ );

- Productivity gains: the internal fluid circulation is also reduced, the trays are less flooded and the feed flowrate can be increased (for the depropanizer, productivity gains are greater than $15 \%$ );

- Process flexibility.

Acknowledgements-The authors are indebted to Professor $H$. Renon for numerous fruitful discussions and to $Y$. Creff for useful suggestions concerning the proof of Lemma 2 and the spectral property of Theorems 1 and 4 . The authors wish also to thank ELF-FRANCE of the group ELFAQUITAINE for supporting this research program, and especially F. Djenab of the Centre de Recherche d'ELF a Solaize, A. Douaud and J. P. Beauchêne of the ELF Refinery of Donges for collaborating in the implementation on the depropanizer. The financial support of the E.E.C. (Non Nuclear Energy R. \& D. Programme) is also gratefully acknowledged.

\section{REFERENCES}

Agarwal, M. and D. E. Seborg (1987). A multivariable nonlinear self-tuning controller. AIChE J., 33, 1376-1386.

Alsop, A. W. and T. F. Edgar (1987). Nonlinear control of a high purity distillation column by the use of partially linearized control variables. AIChE Spring National Meeting, Houston, Texas.

Arnold, V. (1974). Equations Différentielles Ordinaires. Mir, Moscow.

Benallou, A., D. E. Seborg and D. A. Mellichamp (1986). Dynamic compartmental models for separation processes. AIChE J., 32, 1067-1078.

Byrnes, C. I. and A. Isidori (1988). Local stabilization of minimum-phase nonlinear systems. Syst. Control Lett. 11, 9-17.

Duchêne, P. (1988). Simulation dynamique et contrôle d'une cascade de mélangeurs-décanteurs. Rapport de DEA, École des Mines de Paris, France, October 1988.

España, M. and I. D. Landau (1978). Reduced order bilinear models for distillation columns. Automatica, 14, 345355.

Fuentes, C. and W. L. Luyben (1983). Control of high-purity distillation columns. Ind. Engng Chem. Proc. Des. Dev., 22, 361-366.

Gallun, S. E. and C. D. Holland (1982). Gear's procedure for the sinultaneous solution of differential and algebraic equations with applications to unsteady state distillation problems. Computers Chem. Engng, 6, 231-244. 
Gantmacher, F. R. (1966). Théorie des Matrices: Tome 2. Dunod, Paris.

Gauthier, J. P., G. Bornard, S. Bacha and M. Idir (1983). Rejet de Perturbations pour un Modèle Non Linéaire de Colonne à Distiller. Developpement et Utilisation d'Outils et Modèles Mathématiques en Automatique, Analyse des Systèmes et Traitement du Signal, CNRS, Paris.

Georgakis, C. (1986). On the use of extensive variables in process dynamics and control. Chem. Engng Sci., 41, $1471-1484$

Georgiou, A., C. Georgakis and W. L. Luyben (1988). Nonlinear dynamic matrix control for high-purity distillation columns. AIChE J., 34, 1287-1298.

Hirschorn, K. M. (1981). (A, B)-invariant distributions and disturbance decoupling problem of nonlinear systems. SIAM J. Control Optimiz., 19, 1-19.

Hunt, L. R., R. Su and G. Meyer (1983). Design for Multi-input Nonlinear Systems. (Volume 268 of R. W Brockett, R. S. Millman and H. J. Sussman (Eds) Differential Geometric Control Theory).

Isidori, A. (1989). Nonlinear Control Systems. Communications and Control Engineering Series. Springer, Berlin.

Isidori, A., A. Krener, C. Gori-Giorgi and S. Monaco (1981). Nonlinear decoupling via feedback. IEEE Trans. Auto Control, 26, 331-345.

Jakubczyk, B. and W. Respodek (1980). On linearization of control systems. Bull. Acad. Pol. Sci. Ser. Sci. Math., 28, 517-522.

Kokotovic, P. V. (1984). Application of singular perturbation techniques to control problems. SIAM Rev., 26, 501-550.

Krener, A. J. (1984). Approximate linearization by state feedback and coordinate change. Syst. Control Lett. 5, 181-185.

Kummel, M. and H. W. Andersen (1987). Controler adjustment for improved nominal performance and robustness-II: Robust geometric control of distillation column. Chem. Engng Sci. 42, 2011-2023.

Kwaalen, E., L. Neel and D. Tondeur (1985). Directions of quasi-static and energy transfer between phase in multicomponent open systems. Chem. Engng Sci. 40, 1191-1204.

Lévine, J. and P. Rouchon (1986). Disturbances rejection and integral control of aggregated nonlinear distillation models. In Proc. of the 7th Conf. on Analysis and Optimisation of Systems. Lecture Notes in Control and Information Sciences. Springer, Berlin.

Malesinski W. (1965). Azeotropy and Other Theoretical Problems of Vapour-Liquid Equilibrium. Wiley Interscience, New York.

Marino, R. and P. V. Kokotovic (1988). A geometric approach to nonlinear singularly perturbed control systems. Automatica, 24, 31-41.

Morari, M. (1988). Advances in process control theory. Chem. Engng Prog., 84, 60-67.

Prausnitz, J., T. Anderson, E. Grens, C. Eckert, R. Hsieh and J. O'Connell (1980). Computer Calculations for Multicomponent Vapor-Liquid and Liquid-Liquid Equilibria. International Series in the Physical and Chemical Engineering Sciences, Prentice-Hall, Englewood Cliffs, NJ.

Rosenbrock, H. H. (1962). A Liapunov function with applications to some nonlinear physical systems. Automatica, 1, 31-53.

Soave, G. (1972). Equilibrium constants from a modified Redlich-Kwong equation of state. Chem. Engng Sci., 27, 1197-1203.

Speedup User Manual: Release 5.0. (1988). Prosys Technology Cambridge, U.K.

Takamatsu, T., I. Hashimoto and Y. Nakai (1979). A geometric approach to multivariable system design of a distillation column. Automatica, 15, 387-402.

Tikhonov, A., A. Vasil'eva and A. Sveshnikov (1980). Differential Equations. Springer, Berlin, 1980.

Van Winkle, M. (1967). Distillation. McGraw-Hill, 1967.

Wonham, W. M. (1974). Linear Multivariable Control: a Geometric Approach. Springer, Berlin.
APPENDIX A. TWO RESULTS

Rosenbrock (1962) has proven the result given below (Theorem 6 of the appendix).

Rosenbruck's theorem. Consider the differential system of dimension $p>0, \quad \dot{\xi}=\phi(\xi)$. Assume that $\xi=\left(\xi_{i}\right)_{i=1 \ldots, p}$ belongs to $\Omega$, a bounded, closed and convex subset of $\mathbb{R}^{p}$, and that $\phi=\left(\phi_{i}\right)_{i=1, \ldots, p}$ with its partial derivatives are continuous functions of $\xi$. Suppose that:

(i) For each initial condition in $\Omega$, the solution remains in $\Omega$;

(ii) For all $i \in\{1, \ldots, p\}$, the function of $\xi$

$$
\psi_{i}(\xi)=-\sum_{k=1}^{p} \frac{\partial \phi_{k}}{\partial \xi_{i}}(\xi)
$$

is non-negative;

(iii) For all $i$ and $k$ in $\{1, \ldots, p\}$ such that $i \neq k$, $\partial \phi_{k} / \partial \xi_{i} \geq 0$;

(iv) Given any $i \in\{1, \ldots, p\}$ for which $\psi_{i}=0$, there exists $j \neq i$ in $\{1, \ldots, p\}$ such that $\partial \phi_{j} / \partial \xi_{i} \neq 0$; if $\psi_{j}=0$, there exists $k \in\{1, \ldots, p\}$ different of $i$ and $j$ such that $\partial \phi_{k} / \partial \xi_{j} \neq 0$; if $\psi_{k}=0$, then ...; moreover, this process always leads in the end to some $l \in\{1, \ldots, p\}$ for which $\psi_{l} \neq 0$.

Then there exists a unique steady-state in $\Omega$, every solution starting in $\Omega$ converges to this steady-state and the function

$\sum_{k=1}^{p}\left|\phi_{k}(\xi)\right|$ is a Lyapunov function of the system.

We will also use the following lemma.

Lemma 2. Consider $a=\left(a_{i}\right)$ and $b=\left(b_{i}\right)$, two real vectors of dimension $p>0$, and the real $p \times p$ matrix $J=\left(J_{i, j}\right)$ constructed by means of $a$ and $b$ as follows:

- For $i=2, \ldots, p, J_{i, i-1}=a_{i-1}$;

- For $i=1, \ldots, p, J_{i, i}=-a_{i}-b_{i}$

- For $i=1, \ldots, p-1, J_{i, i+1}=b_{i+1}$

- For $i, j=1, \ldots, p$ such that $|i-j|>1, J_{i, j}=0$.

If for all $i \in\{0, \ldots, p\}, a_{i}>0$ and $b_{i}>0$, then the eigenvalues of $J$ are distinct, real and negative.

The proof of this lemma is a straightforward application of a classical result relative to Jacobi's matrix [Gantmacher (1966, p. 99)].

\section{APPENDIX B. THE TIKHONOV THEOREM}

Consider the singularly perturbed system

$$
\begin{aligned}
& \dot{x}^{S}=f^{S}\left(x^{S}, x^{F}, u(t), w(t), \varepsilon\right) \quad x^{S}(0)=x^{S, 0} \\
& \varepsilon \dot{x}^{F}=f^{F}\left(x^{S}, x^{F}, u(t), w(t), \varepsilon\right) \quad x^{F}(0)=x^{F, 0},
\end{aligned}
$$

which admits continuous solution $\left(x^{s}(t, \varepsilon), x^{F}(t, \varepsilon)\right)$ in $[0, T], T>0\left(f^{S}\right.$ and $f^{F}$ are continously differentiable functions). The associated slow subsystem is

$$
\begin{aligned}
\dot{x}^{S} & =f^{S}\left(x^{s}, x^{F}, u(t), w(t), 0\right) \quad x^{s}(0)=x^{s, 0} \\
0 & =f^{F}\left(x^{s}, x^{F}, u(t), w(t), 0\right) .
\end{aligned}
$$

We suppose that it admits a continuous solution $\left(x_{0}^{S}(t), x_{0}^{F}(t)\right)$ in $[0, T]$. For $\vec{t} \in[0, T]$, the associated fast subsystem is

$$
\frac{\mathrm{d} x^{F}}{\mathrm{~d} \tau}=f^{F}\left(x_{0}^{S}(\bar{t}), x^{F}(\tau), u(\bar{t}), w(\bar{t}), 0\right),
$$

where $\tau=t / \varepsilon$.

Tokhonov's theorem. If, for each $\hat{t} \in[0, T]$, the tangent linearization of the fast subsystem around $x_{0}^{F}(\bar{t})$ produces a stable linear system, and if $x^{F, 0}$ belongs to the region of attraction of $x_{0}^{F}(0)$ then

$$
\begin{aligned}
& \lim _{\varepsilon \rightarrow 0^{+}} x^{s}(t, \varepsilon)=x_{0}^{s}(t) \\
& \lim _{\varepsilon \rightarrow 0^{+}} x^{F}(t, \varepsilon)=x_{0}^{F}(t)
\end{aligned}
$$

uniformly on all closed subsets of $] 0, T[$. More details can be found for example in Tikhonov et al. (1980). 